\title{
VIRTUALLY CYCLIC DIMENSION FOR 3-MANIFOLD GROUPS
}

\author{
KYLE JOECKEN, JEAN-FRANÇOIS LAFONT, AND LUIS JORGE SÁNCHEZ SALDAÑA
}

\begin{abstract}
Let $\Gamma$ be the fundamental group of a connected, closed, orientable 3-manifold. We explicitly compute its virtually cyclic geometric dimension $\operatorname{gd}(\Gamma)$. Among the tools we use are the prime and JSJ decompositions of $M$, several push-out type constructions, as well as some Bredon cohomology computations.
\end{abstract}

\section{INTRODUCTION}

Given a group $\Gamma$, we say that a collection of subgroups $\mathcal{F}$ is called a family if it is closed under conjugation and under taking subgroups. We say that a $\Gamma$-CW-complex $X$ is a model for the classifying space $E_{\mathcal{F}} \Gamma$ if every isotropy group of $X$ belongs to $\mathcal{F}$, and $X^{H}$ is contractible whenever $H$ belongs to $\mathcal{F}$. Such a model always exists and it is unique up to $\Gamma$-homotopy equivalence. The geometric dimension of $\Gamma$ with respect to the family $\mathcal{F}$, denoted $\operatorname{gd}_{\mathcal{F}}(\Gamma)$, is the minimum dimension $n$ such that $\Gamma$ admits an $n$-dimensional model for $E_{\mathcal{F}} \Gamma$.

Classical examples of families are the family that consists only of the trivial subgroup $\{1\}$, and the family $F_{I N}$ of finite subgroups of $\Gamma$. A group is said to be virtually cyclic if it contains a cyclic subgroup (finite or infinite) of finite index. We will also consider the family $V_{C Y C}$ of virtually cyclic subgroups of $\Gamma$. These three families are relevant to the Farrell-Jones and the Baum-Connes isomorphism conjectures.

In the present paper we study $\operatorname{gd}_{V C Y C}(\Gamma)$ (also denoted $\operatorname{gd}(\Gamma)$ ) when $\Gamma$ is the fundamental group of an orientable, closed, connected 3-manifold. We call a group non-elementary if it is not virtually cyclic. Our main result is the following theorem.

Theorem 1.1. Let $M$ be a connected, closed, oriented 3-manifold, and let $\Gamma=\pi_{1}(M)$ be the

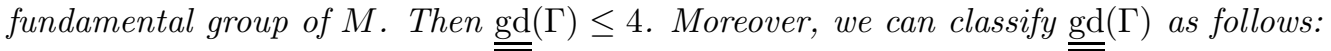

(1) $\operatorname{gd}(\Gamma)=0$ if and only if $\bar{\Gamma}$ is virtually cyclic;

(2) $\overline{\overline{g d}}(\Gamma)=2$ if and only if $\Gamma$ is a non-elementary free product of virtually cyclic groups;

(3) $\overline{\overline{\operatorname{gd}}}(\Gamma)=4$ if and only if $\Gamma$ contains a $\mathbb{Z}^{3}$ subgroup;

(4) $\underline{\underline{\underline{\operatorname{gd}}}}(\Gamma)=3$ in all other cases.

Since we are dealing with 3-manifold groups, the purely group theoretic description given above also corresponds to the following more geometric characterization of the virtually cyclic geometric dimension.

Corollary 1.2. Let $M$ be a connected, closed, oriented 3-manifold, and let $M=P_{1} \# \cdots \# P_{k}$ be the prime decomposition of $M$. Let $\Gamma=\pi_{1}(M)$ be the fundamental group of $M$. Then we can classify

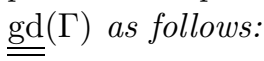

(1) $\underline{\underline{\operatorname{gd}}}(\Gamma)=0$ if and only if $M$ is modeled on $S^{3}$ or $S^{2} \times \mathbb{R}$;

(2) $\overline{\overline{g d}}(\Gamma)=2$ if and only if every $P_{i}$ in the prime decomposition of $M$ is modeled on $S^{3}$ or $\overline{\overline{S^{2}}} \times \mathbb{E}$, and either: (1) $k>2$, or (2) $M=P_{1} \# P_{2}$ with $\left|\pi_{1}\left(P_{1}\right)\right|>2$; 
(3) $\operatorname{gd}(\Gamma)=4$ if and only if at least one of the prime components $P_{i}$ is modeled on $\mathbb{E}^{3}$;

(4) $\underline{\overline{\mathrm{gd}}}(\Gamma)=3$ in all other cases.

Our main tools for proving Theorem 1.1 is the Kneser-Milnor prime decomposition and the Jaco-Shalen-Johannson JSJ decomposition of a 3-manifold, the push-out constructions associated to acylindrical splittings from [LO09b], the theory of Seifert fibered and hyperbolic manifolds, and some Bredon cohomology computations.

The paper is organized as follows. In Section 2 we review the notions of geometric and cohomological dimensions for families of subgroups and some of the basic notation of Bass-Serre theory. Section 3 is devoted to recalling some basics of 3-manifold theory, such as the prime decomposition of Kneser-Milnor and the JSJ decomposition of Jaco-Shalen-Johannson. In Section 4 we state some useful push-out constructions that will help us construct new classifying spaces out of old ones. We also relate the geometric dimension of the fundamental group of a graph of groups with that of the vertex and edges groups, provided that the splitting is acylindrical. This allows us to establish Theorem 4.11 which reduces the calculation to the case of prime manifolds. We then analyze case-by-case the situation when $M$ is a Seifert fibered space (Section 5) or a hyperbolic manifold (Section 6), with the results of these analyses summarized in Tables 1 and 2 , In Section 7 , we focus on 3-manifolds whose JSJ decomposition only contains pieces that are Seifert fibered with Euclidean orbifold base - and show that these manifolds are always geometric. The main result of Section 8 is Theorem 8.1, where for a non-geometric prime 3-manifold, we show that the JSJ decomposition gives rise to an acylindrical splitting. Section 9 then finishes the computation of the virtually cyclic geometric dimension for all the prime manifolds that are not geometric. Finally, in Section 10, we bring these results together and prove Theorem 1.1.

\section{Acknowledgments}

J.-F.L. was partially supported by the NSF, under grant DMS-1812028. L.J.S.S. was supported by the National Council of Science and Technology (CONACyT) of Mexico via the program "Apoyo para Estancias Posdoctorales en el Extranjero Vinculadas a la Consolidación de Grupos de Investigación y Fortalecimiento del Posgrado Nacional". The third author also wants to thank the OSU math department for its hospitality, and specially the second named author.

\section{Preliminaries}

2.1. Virtually cyclic geometric and cohomological dimension. Let $\Gamma$ be a discrete group. A nonempty set $\mathcal{F}$ of subgroups of $\Gamma$ is called a family if it is closed under conjugation and passing to subgroups. We call a $\Gamma$-CW-complex a model for $E_{\mathcal{F}} \Gamma$ if for every $H \leq \Gamma$ :

(1) $H \notin \mathcal{F} \Rightarrow X^{H}=\emptyset\left(X^{H}\right.$ is the $H$-fixed subcomplex of $\left.X\right)$;

(2) $H \in \mathcal{F} \Rightarrow X^{H}$ is contractible.

Such models always exist for every discrete group $\Gamma$ and every family of subgroups $\mathcal{F}$. Moreover, every pair of models for $E_{\mathcal{F}} \Gamma$ are $\Gamma$-homotopically equivalent. The geometric dimension $\operatorname{gd}_{\mathcal{F}}(\Gamma)$ of $\Gamma$ with respect to the family $\mathcal{F}$, is the minimum $n$ for which an $n$-dimensional model for $E_{\mathcal{F}} \Gamma$ exists.

On the other hand, given $\Gamma$ and $\mathcal{F}$ we have the so-called restricted orbit category $\mathcal{O}_{\mathcal{F}} \Gamma$, which has as objects the homogeneous $\Gamma$-spaces $\Gamma / H, H \in \mathcal{F}$, and morphisms consisting of $\Gamma$-maps between them. We define an $\mathcal{O}_{\mathcal{F}} \Gamma$-module to be a functor from $\mathcal{O}_{\mathcal{F}} \Gamma$ to the category of abelian groups, while a morphism between two $\mathcal{O}_{\mathcal{F}} \Gamma$-modules is a natural transformation of the underlying functors. Denote by $\mathcal{O}_{\mathcal{F}} \Gamma$-mod the category of $\mathcal{O}_{\mathcal{F}} \Gamma$-modules, which is an abelian category with enough projectives. 
Thus we can define a $\Gamma$-cohomology theory for $\Gamma$-spaces $H_{\mathcal{F}}^{*}(-; F)$ for every $\mathcal{O}_{\mathcal{F}} \Gamma$-module $F$. The Bredon cohomological dimension of $G$ - denoted $\operatorname{cd}_{\mathcal{F}}(G)$ - is the largest nonnegative $n \in \mathbb{Z}$ for which the Bredon cohomology group $H_{\mathcal{F}}^{n}(G ; F)=H_{\mathcal{F}}^{n}\left(E_{\mathcal{F}} G ; F\right)$ is nontrivial for some $M \in \operatorname{Mod}-\mathcal{O}_{\mathcal{F}} G$.

In the present work we are mainly concerned with the family $V_{C Y C}$ of virtually cyclic subgroups. A highly related family is the family $F_{I N}$ of finite subgroups. We will denote $E_{V C Y C} \Gamma\left(\operatorname{resp} \cdot \operatorname{gd}_{V C Y C}(\Gamma)\right.$, $\left.\operatorname{cd}_{V C Y C}(\Gamma), H_{V C Y C}^{*}\right)$ and $E_{F_{I N}} \Gamma\left(\right.$ resp. $\left.\operatorname{gd}_{F_{I N}}(\Gamma), \operatorname{cd}_{F_{I N}}(\Gamma), H_{F I N}^{*}\right)$ as $\underline{\underline{E}} \Gamma\left(\operatorname{resp} \cdot \underline{\underline{g d}}(\Gamma), \underline{\underline{\operatorname{cd}}}(\Gamma), \underline{\underline{H}}^{*}\right)$ and $\underline{E} \Gamma$ (resp. $\left.\underline{\operatorname{gd}}(\Gamma), \underline{\operatorname{cd}}(\Gamma), \underline{H}^{*}\right)$ respectively. We also call $\underline{\operatorname{gd}}(\Gamma)$ the virtually cyclic (or $\overline{V C}$ ) geometric dimension of $\Gamma$.

Lemma 2.1. We have the following properties of the geometric dimension:

(1) If $H \leq G$, then $\operatorname{gd}_{\mathcal{F} \cap H}(H) \leq \operatorname{gd}_{\mathcal{F}}(G)$ for every family $\mathcal{F}$ of $G$.

(2) For every group $G$ and every family of subgroups $\mathcal{F}$ we have

$$
\operatorname{cd}_{\mathcal{F}}(G) \leq \operatorname{gd}_{\mathcal{F}}(G) \leq \max \left\{3, \operatorname{cd}_{\mathcal{F}}(G)\right\} .
$$

In particular, if $\operatorname{cd}_{\mathcal{F}}(G) \geq 3$, then $\operatorname{cd}_{\mathcal{F}}(G)=\operatorname{gd}_{\mathcal{F}}(G)$.

(3) If $G$ is an $n$-crystallographic group then $\operatorname{gd} G=n+1$

Proof. Statement (1) Follows from the observation that a model for $E_{\mathcal{F}} G$ is also a model for $E_{H \cap \mathcal{F}} H$, just by restricting the $G$-action to the subgroup $H \leq G$. Statement (2) is the main result of [LM00], while statement (3) follows from [CFH06.

2.2. Graphs of groups. In this subsection we give a quick review of Bass-Serre theory, referring the reader to [Ser03 for more details. A graph (in the sense of Bass and Serre) consists of a set of vertices $V=\operatorname{vert} Y$, a set of (oriented) edges $E=\operatorname{edge} Y$, and two maps $E \rightarrow V \times V, y \mapsto(o(y), t(y))$, and $E \rightarrow E, y \mapsto \bar{y}$ satisfying $\overline{\bar{y}}=y, \bar{y} \neq y$, and $o(y)=t(\bar{y})$. The vertex $o(y)$ is called the origin of $y$, and the vertex $t(y)$ is called the terminus of $y$.

An orientation of a graph $Y$ is a subset $E_{+}$of $E$ such that $E=E_{+} \sqcup \bar{E}_{+}$. We can define path and circuit in the obvious way.

A graph of groups $\mathbf{Y}$ consists of a graph $Y$, a group $Y_{P}$ for each $P \in \operatorname{vert} Y$, and a group $Y_{y}$ for each $y \in$ edge $Y$, together with monomorphisms $Y_{y} \rightarrow Y_{t(y)}$. One requires in addition $Y_{\bar{y}}=Y_{y}$.

Suppose that the group $G$ acts without inversions on a graph $X$, i.e. for every $g \in G$ and $x \in$ edge $X$ we have $g x \neq \bar{x}$. Then we have an induced graph of groups with underlying graph $X / G$ by associating to each vertex (resp. edge) the isotropy group of a preimage under the quotient map $X \rightarrow X / G$.

Given a graph of groups $\mathbf{Y}$, one of the classic theorems of Bass-Serre theory provides the existence of a group $G=\pi_{1}(\mathbf{Y})$, called the fundamental group of the graph of groups $\mathbf{Y}$ and a tree $T$ (a graph with no cycles), called the Bass-Serre tree of $\mathbf{Y}$, such that $G$ acts on $T$ and the induced graph of groups is isomorphic to $\mathbf{Y}$. The identification $G=\pi_{1}(\mathbf{Y})$ is called a splitting of $G$.

Analogously we can define a graph of spaces $\mathbf{X}$ as a graph $X, \mathrm{CW}$-complexes $X_{P}$ and $X_{y}$ for each vertex $P$ and each edge $y$, and closed cellular embeddings $X_{y} \rightarrow X_{P}$ if either $P=t(y)$ or $P=o(y)$. We also assume that the images of the embeddings are disjoint. In this case we will have a CW-complex, called the geometric realization, that is assembled by gluing the ends of the product space $X_{y} \times[0,1]$ to the spaces $X_{t(y)}, X_{o(y)}$.

Finally, given a graph of spaces $\mathbf{X}$ with $\pi_{1}\left(X_{y}\right) \rightarrow \pi_{1}\left(X_{P}\right)$ injective, there is an associated graph of groups $\mathbf{Y}$ with the same underlying graph and whose vertex (resp. edge) groups are the fundamental groups of the corresponding vertex (resp. edges) CW-complexes. Then, as a generalization of the Seifert-van Kampen theorem, we have that the fundamental group of the geometric realization of $\mathbf{X}$ is naturally isomorphic to the fundamental group of the graph of groups $\mathbf{Y}$. 


\section{3-MANIFOLDS AND DECOMPOSITIONS}

In this section we will review some 3-manifold theory. For more details see [Sc083, Mor05.

3.1. Seifert fibered spaces. A trivial fibered solid torus is the usual product $S^{1} \times D^{2}$ with the product foliation by circles $S^{1} \times\{y\}, y \in D^{2}$. A fibered solid torus is a solid torus with a foliation by circles which is finitely covered by a trivial fibered solid torus. Similarly, a fibered solid Klein bottle is a solid Klein bottle which is finitely covered by a trivial fibered solid torus.

A Seifert fiber space is a 3-manifold with a decomposition into disjoint circles, called fibers, such that each circle has a neighborhood which is a union of fibers and is isomorphic to a fibered solid torus or a fibered Klein bottle.

Given a Seifert fiber space $M$, one can obtain an orbifold $B$ by quotienting out by the $S^{1}$-action on the fibers of $M$; that is, by identifying each fiber to a point. By considering the quotient of neighborhoods of fibers in $M$, the topology $B$ inherits makes it a surface with a natural orbifold structure; we call $B$ the base orbifold of $M$. Such an orbifold $B$ has its orbifold fundamental group, which is not necessarily the fundamental group of the underlying topological space, but is related to the fundamental group of $M$ via the following lemma.

Lemma 3.1. Sco83, Lemma 3.2] Let $M$ be a Seifert fiber space with base orbifold B. Let $\Gamma$ be the fundamental group of $M$, and let $\Gamma_{0}$ be the orbifold fundamental group of $B$. Then there is an exact sequence

$$
1 \rightarrow K \rightarrow \Gamma \rightarrow \Gamma_{0} \rightarrow 1
$$

where $K$ denotes the cyclic subgroup of $\Gamma$ generated by a regular fiber. The group $K$ is infinite except in cases where $M$ is covered by $S^{3}$.

Recall that an orbifold is called good if it is the quotient of a manifold by an action of a discrete group of isometries. An orbifold that is not good is called bad.

It is known that every good 2-orbifold is isomorphic, as an orbifold, to the quotient of $S^{2}, \mathbb{E}^{2}$, or $\mathbb{H}^{2}$ by some discrete subgroup of isometries. Hence all closed good 2-orbifolds can be classified as spherical, euclidean or flat, and hyperbolic. Bad 2-orbifolds are classified in [Sco83, Theorem 2.3].

3.2. Geometric 3-manifolds. A Riemannian manifold $X$ is a smooth manifold that admits a Riemannian metric. If the isometry group $\operatorname{Isom}(X)$ acts transitively, we say $X$ is homogeneous. If in addition $X$ has a quotient of finite volume, $X$ is unimodular. A geometry is a simply-connected, homogeneous, unimodular Riemannian manifold along with its isometry group. Two geometries $(X, \operatorname{Isom}(X))$ and $\left(X^{\prime}, \operatorname{Isom}\left(X^{\prime}\right)\right)$ are equivalent if $\operatorname{Isom}(X) \cong \operatorname{Isom}\left(X^{\prime}\right)$ and there exists a diffeomorphism $X \rightarrow X^{\prime}$ that respects the $\operatorname{Isom}(X)$, $\operatorname{Isom}\left(X^{\prime}\right)$ actions. A geometry $(X, \operatorname{Isom}(X))$ (often abbreviated $X$ ) is maximal if there is no Riemannian metric on $X$ with respect to which the isometry group strictly contains $\operatorname{Isom}(X)$. A manifold $M$ is called geometric if there is a geometry $X$ and discrete subgroup $\Gamma \leq \operatorname{Isom}(X)$ with free $\Gamma$-action on $X$ such that $M$ is diffeomorphic to the quotient $X / \Gamma$; we also say that $M$ admits a geometric structure modeled on $X$. Similarly, a manifold with nonempty boundary is geometric if its interior is geometric.

It is a consequence of the uniformization theorem that compact surfaces (2-manifolds) admit Riemannian metrics with constant curvature; that is, compact surfaces admit geometric structures modeled on $\mathbb{S}^{2}, \mathbb{E}^{2}$, or $\mathbb{H}^{2}$. In dimension three, we are not guaranteed constant curvature. Thurston demonstrated that there are eight 3-dimensional maximal geometries up to equivalence ( $\mathrm{Sco83}$, Theorem 5.1]): $\mathbb{S}^{3}, \mathbb{E}^{3}, \mathbb{H}^{3}, \mathbb{S}^{2} \times \mathbb{E}, \mathbb{H}^{2} \times \mathbb{E}, \widetilde{\mathrm{PSL}}_{2}(\mathbb{R})$, Nil, and Sol. 
3.3. Prime and JSJ decomposition. A closed $n$-manifold is an $n$-manifold that is compact with empty boundary. A connected sum of two $n$-manifolds $M$ and $N$, denoted $M \# N$, is a manifold created by removing the interiors of a smooth $n$-disc $D^{n}$ from each manifold, then identifying the boundaries $\mathbb{S}^{n-1}$. An $n$-manifold is nontrivial if it is not homeomorphic to $\mathbb{S}^{n}$. A prime $n$-manifold is a nontrivial manifold that cannot be decomposed as a connected sum of two nontrivial $n$-manifolds; that is, $M=N \# P$ for some $n$-manifolds $N, P$ forces either $N=\mathbb{S}^{n}$ or $P=\mathbb{S}^{n}$. An $n$-manifold $M$ is called irreducible if every 2-sphere $\mathbb{S}^{2} \subset M$ bounds a ball $D^{3} \subset M$. It is well-known that all orientable prime manifolds are irreducible with the exception of $S^{1} \times S^{2}$. The following is a well-known theorem of Kneser (existence) and Milnor (uniqueness).

Theorem 3.2 (Prime decomposition). Let $M$ be a closed oriented nontrivial 3-manifold. Then $M=P_{1} \# \ldots \# P_{n}$ where each $P_{i}$ is prime. Furthermore, this decomposition is unique up to order and homeomorphism.

Another well known result we will need is the Jaco-Shalen-Johannson decomposition, after Perelman's work.

Theorem 3.3 (JSJ decomposition). For a closed, prime, oriented 3-manifold $M$ there exists a collection $T \subseteq M$ of disjoint incompressible tori, i.e. two sided properly embedded and $\pi_{1}$-injective, such that each component of $M \backslash T$ is either a hyperbolic or a Seifert fibered manifold. A minimal such collection $T$ is unique up to isotopy.

Remark 3.4. Note that the prime decomposition provides a graph of groups with trivial edge groups and vertex groups isomorphic to the fundamental group of the $P_{i}$ 's. The fundamental group of the graph of groups will be isomorphic to $\pi_{1}(M)$. Similarly the JSJ decomposition of a prime 3 -manifold $M$ gives rise to a graph of groups, with all edge groups isomorphic to $\mathbb{Z}^{2}$, and vertex groups isomorphic to the fundamental groups of the Seifert fibered and hyperbolic pieces. Again, the fundamental group of the graph of groups will be isomorphic to $\pi_{1}(M)$. Each graph of groups provide a splitting for the fundamental groups of the initial manifold. These splittings will be used to provide reductions of the general computation to some special cases.

\section{Push-out COnstructions for Classifying SPACES}

In this section we will review some push-out constructions, used to construct new classifying spaces out of old (or known) ones.

Definition 4.1. Let $\Gamma$ be any finitely generated group, and $\mathcal{F} \subset \mathcal{F}^{\prime}$ a pair of families of subgroups of $\Gamma$. We say a collection $\mathcal{A}=\left\{A_{\alpha}\right\}_{\alpha \in I}$ of subgroups of $\Gamma$ is adapted to the pair $\left(\mathcal{F}, \mathcal{F}^{\prime}\right)$ provided that the following conditions hold:

(1) For all $A, B \in \mathcal{A}$, either $A=B$ or $A \cap B \in \mathcal{F}$;

(2) The collection $\mathcal{A}$ is conjugacy closed;

(3) Every $A \in \mathcal{A}$ is self normalizing; i.e. $N_{\Gamma}(A)=A$;

(4) For all $A \in \mathcal{F}^{\prime} \backslash \mathcal{F}$, there is a $B \in \mathcal{A}$ such that $A \leq B$.

Proposition 4.2. [LO09a, p. 302] Let $\mathcal{F} \subset \mathcal{F}^{\prime}$ be families of subgroup of $\Gamma$. Assume that the collection of subgroups $\mathcal{A}=\left\{H_{\alpha}\right\}_{\alpha \in I}$ is adapted to the pair $\left(\mathcal{F}, \mathcal{F}^{\prime}\right)$. Let $\mathcal{H}$ be a complete set of representatives of the conjugacy classes within $\mathcal{A}$, and consider the cellular $\Gamma$-push-out

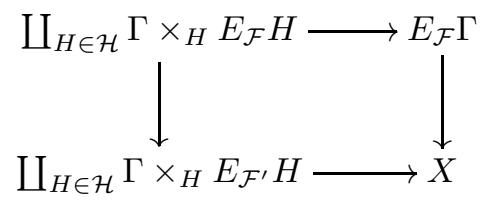


Then $X$ is a model for $E_{\mathcal{F}^{\prime}} \Gamma$. In the above cellular $\Gamma$-push-out we require either (1) the left vertical map is the disjoint union of cellular $H$-maps, and the upper horizontal is an inclusion of $\Gamma-C W$ complexes, or (2) the right vertical map is the disjoint union of inclusion of $H$-CW-complexes, and the upper horizontal map is a cellular $\Gamma$-map.

The following lemmas are straightforward consequences of the definition of adapted collections.

Lemma 4.3. Let $\Gamma$ be a finitely generated discrete group, and let $\mathcal{F} \subseteq \mathcal{F}^{\prime} \subseteq \mathcal{F}^{\prime \prime}$ be three nested families of subgroups of $\Gamma$. Let $\mathcal{A}$ be a collection adapted to the pair $\left(\mathcal{F}, \mathcal{F}^{\prime \prime}\right)$. Then $\mathcal{A}$ is adapted to the pairs $\left(\mathcal{F}, \mathcal{F}^{\prime}\right)$ and $\left(\mathcal{F}^{\prime}, \mathcal{F}^{\prime \prime}\right)$

Lemma 4.4. Let $\varphi: \Gamma \rightarrow \Gamma_{0}$ be surjective group homomorphism of discrete groups, let $\mathcal{F} \subseteq \mathcal{F}^{\prime}$ be pair of families of subgroups of $\Gamma_{0}$, and let $\mathcal{A}=\left\{A_{\alpha}\right\}_{\alpha \in I}$ be a collection adapted to the pair $\left(\mathcal{F}, \mathcal{F}^{\prime}\right)$. Then $\tilde{\mathcal{A}}=\left\{\varphi^{-1}\left(A_{\alpha}\right)\right\}_{\alpha \in I}$ is a collection adapted to the pair $\left(\tilde{\mathcal{F}}, \tilde{\mathcal{F}}^{\prime}\right)$ of families of subgroups of $\Gamma$.

Theorem 4.5. Let $\mathcal{F}$ be a family of subgroups of the finitely generated discrete group $\Gamma$. Let $\varphi: \Gamma \rightarrow \Gamma_{0}$ be a surjective homomorphism. Let $\mathcal{F}_{0} \subseteq \mathcal{F}_{0}^{\prime}$ be a nested pair of families of subgroups of $\Gamma_{0}$ satisfying $\tilde{\mathcal{F}}_{0} \subseteq \mathcal{F} \subseteq \tilde{\mathcal{F}}_{0}^{\prime}$, and let $\mathcal{A}=\left\{A_{\alpha}\right\}_{\alpha \in I}$ be a collection adapted to the pair $\mathcal{F}_{0} \subseteq \mathcal{F}_{0}^{\prime}$. Let $\mathcal{H}$ be a complete set of representatives of the conjugacy classes within $\tilde{\mathcal{A}}=\left\{\varphi^{-1}\left(A_{\alpha}\right)\right\}_{\alpha \in I}$, and consider the following cellular $\Gamma$-push-out

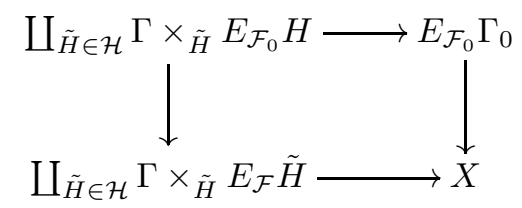

Then $X$ is a model for $E_{\mathcal{F}} \Gamma$. In the above cellular $\Gamma$-push-out we require either (1) the left vertical map is the disjoint union of cellular $\tilde{H}$-maps, and the upper horizontal is an inclusion of $\Gamma-C W$ complexes, or (2) the right vertical map is the disjoint union of inclusion of $\tilde{H}-C W$-complexes, and the upper horizontal map is a cellular $\Gamma$-map.

Proof. It can be easily verified that, via restriction with $\varphi, E_{\mathcal{F}_{0}} \Gamma_{0}=E_{\tilde{\mathcal{F}}_{0}} \Gamma$ and $E_{\mathcal{F}_{0}} H=E_{\tilde{\mathcal{F}}_{0}} \tilde{H}$. From Lemmas 4.3 and 4.4 we have that $\tilde{\mathcal{A}}$ is a collection adapted to the pair $\left(\tilde{\mathcal{F}}_{0}, \mathcal{F}\right)$. Then by Proposition 4.2 we have that the above push-out is a model for $E_{\mathcal{F}} \Gamma$.

The following immediate corollary is more suitable for our purposes, and it will be used jointly with Lemma 3.1

Corollary 4.6. Let $\Gamma$ be finitely generated discrete group. Let $\varphi: \Gamma \rightarrow \Gamma_{0}$ be a surjective homomorphism with cyclic kernel. Let $F_{I N_{0}}$ and $V_{C Y C_{0}}$ be the famlies of finite and virtually cyclic subgroups of $\Gamma_{0}$ respectively. Let $\mathcal{A}$ be a collection adapted to the pair $\left(F_{I N_{0}}, V_{C Y C_{0}}\right)$. Let $\mathcal{H}$ be a complete set of representatives of the conjugacy classes within $\tilde{\mathcal{A}}$, and consider the following cellular $\Gamma$-push-out

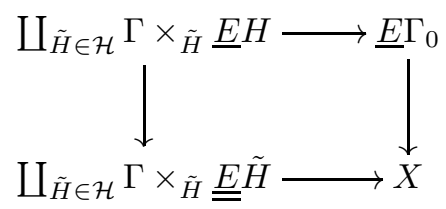

Then $X$ is a model for $\underline{\underline{E}} \Gamma$. In the above cellular $\Gamma$-push-out we require either (1) the left vertical map is the disjoint union of cellular $\tilde{H}$-maps, and the upper horizontal is an inclusion of $\Gamma-C W$ complexes, or (2) the right vertical map is the disjoint union of inclusion of $\tilde{H}-C W$-complexes, and the upper horizontal map is a cellular $\Gamma$-map. 
Next, we will show how to construct a classifying space (for a suitable family) of the fundamental group of a graph of groups, by assembling the classifying spaces of the vertex groups and the edge groups. We will later apply these constructions in conjunction with Remark 3.4.

Let $\mathbf{Y}$ be a graph of groups with vertex groups $Y_{P}$ and edge groups $Y_{y}$, and fundamental group $G$. We are going to construct a graph of spaces $\mathbf{X}$ using the classifying spaces of the edges and the vertices and the corresponding families of virtually cyclic subgroups. Let $X_{P}$ be a model for for the classifying space $\underline{\underline{E}} Y_{P}$, and $X_{y}$ be a model for $\underline{\underline{E}} Y_{y}$, for every vertex $P$ and edge $y$ of $Y$. So for every monomorphism $\overline{\bar{Y}}_{y} \rightarrow Y_{t(y)}$ we have a $Y_{y}$-equivariant cellular map (unique up to $Y_{y}$-homotopy) $X_{y} \rightarrow X_{t(y)}$ which leads to the $G$-equivariant cellular map $G \times_{Y_{y}} X_{y} \rightarrow G \times_{Y_{t(y)}} X_{t(y)}$. This gives us the information required to define a graph of spaces $\mathbf{X}$ with underlying graph $T$, the Bass-Serre tree of $\mathbf{Y}$. Moreover, we have a cellular $G$-action on the geometric realization $X$ of $\mathbf{X}$.

Proposition 4.7. The geometric realization $X$ of the graph of spaces $\mathbf{X}$ constructed above is a model for $E_{\mathcal{F}} G$, where $\mathcal{F}$ is the family of virtually cyclic subgroups of $G$ that are conjugate to a virtually cyclic subgroup in one of the $Y_{y}$ or $Y_{P}, y \in \operatorname{edge} Y, P \in \operatorname{vert} Y$. In particular, there exists a model for $E_{\mathcal{F}} G$ of dimension

$$
\operatorname{gd}_{\mathcal{F}}(G) \leq \max \left\{\underline{\underline{\operatorname{gd}}}\left(Y_{y}\right)+1, \underline{\underline{\operatorname{gd}}}\left(Y_{P}\right) \mid y \in \operatorname{edge} Y, P \in \operatorname{vert} Y\right\} .
$$

Proof. Let $\phi: X \rightarrow T$ be a deformation retraction collapsing each copy of $X_{P}$ down onto the vertex $\widetilde{P} \in \operatorname{vert} T$ to which it corresponds, and similarly collapsing each $X_{y} \times[0,1]$ down along the $X_{y}$ component to the corresponding edge $\widetilde{y} \in \operatorname{edge} T, \widetilde{y} \simeq[0,1]$. Then $G$ has a natural action on $X$ via left multiplication, which permutes the copies of each $X_{P}$ or $X_{y}$ so that $\phi$ is a $G$-equivariant map. It remains to show that the $G-C W$-complex $X$ is a model for $E_{\mathcal{F}} G$.

Suppose first that $H \leq G$ is not in $\mathcal{F}$; then either $H$ is not conjugate into any vertex subgroup or is not virtually cyclic. If $H$ is not conjugate into any vertex subgroup, then $T^{H}=\emptyset$; in particular, $H$ does not fix any copy of $X_{P}$ or $X_{y} \times[0,1]$ in $X$, so $X^{H}=\emptyset$. If $H$ is not virtually cyclic, then even if the $H$-action on $T$ does fix some nonempty subgraph, the $H$-action on any corresponding $X_{P}$ or $X_{y} \times[0,1]$ must have empty fixed set; again, this implies that $X^{H}=\emptyset$.

On the other hand, suppose $H \in \mathcal{F}$; that is, $H$ is virtually cyclic and conjugate into the subgroup $Y_{P}$, for some vertex $P$. Then $H$ fixes the copy of $X_{P}$ in $X$ corresponding to a fixed vertex in $T$. As $H$ is virtually cyclic, and $X_{P}$ is a model for $\underline{E} Y_{P},\left(X_{P}\right)^{H}$ is not empty. Moreover, given any two vertices $P$ and $Q$ in vert $T$ fixed by the $H$-action, the unique geodesic path $c$ in $T$ connecting $P$ and $Q$ must also be fixed; in particular, $T^{H}$ is a connected subgraph of the tree $T$, so that $T^{H}$ is itself a tree. Let $\widetilde{y} \in$ edge $T$ be fixed, and consider the copy $X_{y} \times[0,1]$ with $\phi$-image $\widetilde{y}$; then the $H$-action on $X_{y}$ has nonempty fixed set $\left(X_{y}\right)^{H}$, so in particular $\left(X_{y}\right)^{H} \times[0,1]$ is nonempty. Thus, the $\phi$-preimage of $T^{H}$ is a nonempty, connected subspace $X^{H} \subseteq X$. To see that $X^{H}$ is contractible, first contract down along $\phi$ to $T^{H}$, then contract the tree $T^{H}$.

Definition 4.8. Let $\mathbf{Y}$ be a graph of groups with fundamental group $G$. The splitting of $G$ is said to be acylindrical if there exists an integer $k$ such that, for every path $c$ of length $k$ in the Bass-Serre tree $T$ of $\mathbf{Y}$, the stabilizer of $c$ is finite.

The following proposition roughly says that, provided $\mathbf{Y}$ gives an ayclindrical splitting of $G$, you can attach 2-cells to the classifying space from Proposition 4.7 to get a model for $\underline{\underline{E}} G$.

Proposition 4.9. Let $\mathbf{Y}$ be a graph of groups giving an acylindrical splitting of $G$. Let $\mathcal{F}$ be the family of virtually cyclic subgroups of $G$ that conjugate into a vertex group in $\mathbf{Y}$. Let $\mathcal{A}$ be the collection of maximal virtually cyclic subgroups of $G$ not in $\mathcal{F}$. Let $\mathcal{H}$ be a complete set of 
representatives of the conjugacy classes within $\mathcal{A}$. Let $\{*\}$ be the one point space, and consider the following cellular G-push-out

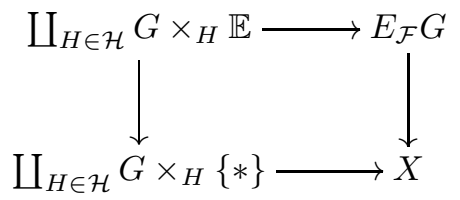

Then $X$ is a model for $\underline{E} G$. In the above cellular $G$-push-out we require either (1) the left vertical map is the disjoint union of cellular $H$-maps, and the upper horizontal is an inclusion of $G-C W$ complexes, or (2) the right vertical map is the disjoint union of inclusion of $\mathrm{H}$-CW-complexes, and the upper horizontal map is a cellular G-map.

Proof. From [LO09b, Claim 3] we know that $\mathcal{A}$ is an adapted collection to the pair $\left(\mathcal{F}, V_{C Y C}\right)$. Hence by Proposition 4.2 we just have to prove that, for all $H \in \mathcal{H}, \mathbb{E}$ and $\{*\}$ are models for $E_{\mathcal{F}} H$ and $\underline{\underline{E}} H$ respectively. It is clear that $\{*\}$ is a model for $\underline{\underline{E}} H$ since $H$ is virtually cyclic. To verify the other case, it suffices to check that $\mathcal{F} \cap H$ is the family of finite subgroups of $H$.

Let $V \in \mathcal{F}$ satisfy $V \leq H$. Then $V$ can be conjugated into $Y_{P}$ for some vertex group in $Y$; say $g V g^{-1} \leq Y_{P}$. Since $V$ is virtually cyclic, let $\langle v\rangle$ be a finite-index cyclic subgroup of $V$. If $\langle v\rangle$ is infinite, then $[H:\langle v\rangle]\left\langle\infty\right.$. If $h \in H \backslash\langle v\rangle$, then $h^{n} \in\langle v\rangle$ for some $n \in \mathbb{N}$, which means that $g h^{n} g^{-1} \in Y_{P}$. Now recall that $G$ can be identified with a subgroup of the free product $F(\mathbf{Y})=*_{\text {vert } Y} Y_{P} * F_{\text {edge } Y}$, where $F_{\text {edge } Y}$ is the free group on edge $Y$. Therefore there is no element $f \in F(\mathbf{Y}) \backslash Y_{P}$ with a power in $Y_{P}$. Thus, we must have that $g h g^{-1} \in Y_{P}$. But this implies that $g H g^{-1} \leq Y_{P}$, contradicting that $H \notin \mathcal{F}$. So we conclude that $\langle v\rangle$ must be finite. Since $V$ has a finite subgroup of finite index, it is also finite.

Conversely, if $F \leq H$ is a finite subgroup, then $T^{F}$ is nonempty (note that $T$ is $\operatorname{CAT}(0)$ ). In particular, $F$ fixes some vertex $g Y_{P} \in \operatorname{vert} T$, and is therefore conjugate to a subgroup of $Y_{P}$. This shows $F \in \mathcal{F}$, giving the reverse containment.

Corollary 4.10. Let $\mathbf{Y}$ be a graph of groups giving an acylindrical splitting of $G$. Then

$$
\underline{\underline{\operatorname{gd}}}(G) \leq \max \left\{2, \underline{\underline{\operatorname{gd}}}\left(Y_{y}\right)+1, \underline{\underline{\operatorname{gd}}}\left(Y_{P}\right) \mid y \in \operatorname{edge} Y, P \in \operatorname{vert} Y\right\} .
$$

Proof. Fix minimal models for $\underline{\underline{E}} Y_{y}$, and $\underline{\underline{E}} Y_{P}$ for every edge $y$ and every vertex $P$ of $Y$. Now from Proposition 4.7 we get a model $X$ for $E_{\mathcal{F}} G$ of dimension $\max \left\{\underline{\underline{\operatorname{gd}}}\left(Y_{y}\right)+1, \underline{\underline{\operatorname{gd}}}\left(Y_{P}\right) \mid y \in \operatorname{edge} Y, P \in\right.$ vert $Y$ \}. Now using Proposition 4.9 we can attach 2-cells to $X$ in order to obtain a model for $\underline{\underline{E}} G$ of

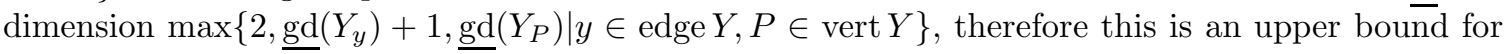

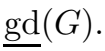

As a quick application, we now explain how to reduce our analysis of the virtually cyclic geometric dimension of a 3-manifold group to the prime case.

Theorem 4.11. Let $M$ be a closed, orientable, connected 3-manifold. Consider the prime decomposition $M=P_{1} \# \cdots \# P_{k}$. Denote $\Gamma=\pi_{1}(M), \Gamma_{i}=\pi_{1}\left(P_{i}\right)$. Then

$$
\max \left\{\underline{\underline{\operatorname{gd}}}\left(\Gamma_{i}\right) \mid 1 \leq i \leq k\right\} \leq \underline{\underline{\operatorname{gd}}}(\Gamma) \leq \max \left\{2, \underline{\underline{\operatorname{gd}}}\left(\Gamma_{i}\right) \mid 1 \leq i \leq k\right\} .
$$

Proof. Since each $\Gamma_{i}$ is a subgroup of $\Gamma$, the first inequality comes from Lemma 2.1. On the other hand, the prime decomposition of $M$ determines a graph of spaces with fundamental group $\Gamma$ and hence a graph of groups $\mathbf{Y}$ (see Remark 3.4). Since the edge groups are all isomorphic to $\pi_{1}\left(S^{2}\right)=1$, stabilizers of the edges in the Bass-Serre tree $T$ of $\mathbf{Y}$ are trivial. Thus the splitting of $\Gamma$ is acylindrical (with $k=1$ in Definition 4.8). The conclusion now follows from Corollary 4.10, 
The reader might naturally wonder whether a similar reduction can be performed with the JSJ decomposition. This is indeed the case, but acylindricity of the splitting is much more subtle in that case (and does not always hold). We will discuss this is detail in Section 8.

\section{The SEIFERT FIBERED CASE}

In this section, we will study the geometric dimension of the fundamental groups of compact Seifert fibered manifolds, both in the case where they have toral boundary components (e.g. pieces in the JSJ decomposition of a prime 3-manifold) and the case where they have no boundary (e.g. are themselves prime 3-manifolds).

5.1. Seifert fibered manifolds without boundary. Using the base orbifold $B$ of a Seifert fibered manifold, we have the following classification

- $B$ is a bad orbifold;

- $B$ is a good orbifold, modeled on either $S^{2}, \mathbb{H}^{2}$ or $\mathbb{E}^{2}$.

The following proposition deals with the case where $B$ is a bad manifold, or is a good manifold modeled on $S^{2}$.

Proposition 5.1. Let $M$ be a closed Seifert fiber space with base orbifold $B$ and fundamental group $\Gamma$. Assume that $B$ is either a bad orbifold, or a good orbifold modeled on $S^{2}$. Then $\Gamma$ is virtually cyclic. In particular, $\operatorname{gd}(\Gamma)=0$

Proof. Suppose first that $B$ is modeled on $S^{2}$. Then $\Gamma_{0}=\pi_{1}(B)$ is a discrete subgroup of $S O(3) \cong$ $\operatorname{Isom}\left(S^{2}\right)$ and is therefore finite. By the short exact sequence given in Lemma 3.1 is virtually cyclic.

From the classification of bad manifolds (see Sco83. Theorem 2.3]) we know that if $B$ is a bad manifold then its orbifold Euler characteristic is positive. So by [Sco83, Theorem 5.3(ii)] $M$ is modeled on one of $S^{3}$ or $S^{2} \times \mathbb{E}$. Now we conclude by observing that discrete subgroups in either $\operatorname{Isom}\left(S^{3}\right) \cong S O(4)$ or $\operatorname{Isom}\left(S^{2} \times \mathbb{E}\right) \cong S O(3) \times\left(\mathbb{R} \rtimes \mathbb{Z}_{2}\right)$ are virtually cyclic.

Now we have to deal with the case where $B$ is a good orbifold modeled on $\mathbb{H}^{2}$ or $\mathbb{E}^{2}$.

Proposition 5.2. Let $M$ be a closed Seifert fiber space with base orbifold $B$ modeled on $\mathbb{H}^{2}$. Let $\Gamma=\pi_{1}(M)$ and $\Gamma_{0}=\pi_{1}(B)$ be the respective fundamental groups. Let $\mathcal{A}$ be the collection of maximal infinite virtually cyclic subgroups of $\Gamma_{0}$, let $\widetilde{\mathcal{A}}$ be the collection of preimages of $\mathcal{A}$ in $\Gamma$, and let $\mathcal{H}$ be a set of representatives of conjugacy classes in $\widetilde{\mathcal{A}}$. Consider the following cellular $\Gamma$-push-out:

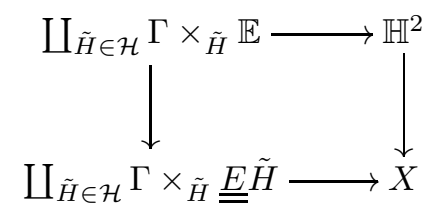

Then all $\widetilde{H} \in \mathcal{H}$ are virtually 2-crystallographic, and $X$ is a model for $\underline{\underline{E}} \Gamma$. In the above cellular $\Gamma$-push-out, we require either (1) the left vertical map is the disjoint union of cellular $\widetilde{H}$-maps $(\widetilde{H} \in \mathcal{H})$, the upper horizontal map is an inclusion of $\Gamma$-CW-complexes, or (2) the left vertical map is the disjoint union of inclusions of $\widetilde{H}-C W$-complexes $(\widetilde{H} \in \mathcal{H})$, the upper horizontal map is a cellular $\Gamma$-map.

Proposition 5.3. Let $M$ be a closed Seifert fibered manifold with base orbifold modeled on $\mathbb{H}^{2}$, and

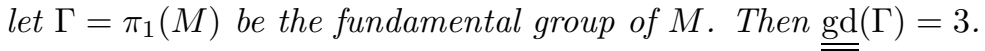


Proof of Proposition 5.2. We have the short exact sequence

$$
1 \rightarrow \mathbb{Z} \rightarrow \Gamma \rightarrow \Gamma_{0} \rightarrow 1
$$

from Lemma 3.1. Then $\Gamma_{0}$ is a lattice in $\operatorname{Isom}\left(\mathbb{H}^{2}\right) \cong \mathrm{PSL}_{2}(\mathbb{R}) \rtimes \mathbb{Z} / 2$, hence is hyperbolic. Let $V_{C Y C_{0}}$ and $F_{I N_{0}}$ be the families of virtually cyclic subgroups and finite subgroups of $\Gamma_{0}$ respectively. Applying [LO07, Theorem 2.6], we see that the collection $\mathcal{A}$ of maximal infinite virtually cyclic subgroups of $\Gamma_{0}$ is adapted to the pair $\left(F_{I N_{0}}, V_{C Y C_{0}}\right)$.

This allows us to apply the construction of Corollary 4.6. Since $\Gamma_{0}$ is a lattice in Iso( $\left(\mathbb{H}^{2}\right)$, we have that $\mathbb{H}^{2}$ is a model for $\underline{E} \Gamma_{0}$. Let $H \in \mathcal{A}$. Therefore $H$ is the stabilizer of a unique geodesic $c$ in $\mathbb{H}^{2}$. The subgroup of $H$ that fixes $c$ is finite and normal; the quotient of $H$ by this group inherits an effective action on $c \simeq \mathbb{E}$, and is therefore 1-crystallographic. This gives $\mathbb{E}$ as a model for $\underline{E} H$.

Consider $\widetilde{H} \in \mathcal{H}, H=\phi(\widetilde{H})$ and its action on $\mathbb{H}^{2}$ (on which $B$ is modeled). As $H$ is virtually cyclic, we know it stabilizes a unique geodesic $c$, hence $H$ has a natural action on $\mathbb{E}$ as $c \simeq \mathbb{E}$. We now consider the preimage of this copy of $\mathbb{E}$ in the lift of the Seifert fiber space $M$ to its universal cover. By Lemma 3.1, $\Gamma \rightarrow \Gamma_{0}$ is infinite cyclic unless $M$ is modeled on $S^{3}$, but this will contradict Sco83, Theorem 5.3]; lifting $\mathbb{E}$ to $\widetilde{M}$ we then get a $\widetilde{H}$-action on $\mathbb{E}^{2}$. Let $\widetilde{F} \leq \widetilde{H}$ be the subgroup with trivial action on $\mathbb{E}^{2}$. Then $F=\phi(\widetilde{F})$ is a subgroup $F \leq H$, which cannot contain the hyperbolic element that generates the finite-index infinite cyclic subgroup of $H$, so must be finite. Since ker $\phi \cong \mathbb{Z}$ acts non-trivially on the fiber direction, $\widetilde{F} \cong F$ must also be finite. Letting $Q=\widetilde{H} / \widetilde{F}$, we get that $Q$ is 2-crystallographic, as it inherits an effective cocompact action on $\mathbb{E}^{2}$. In particular the model for $\underline{\underline{E}} Q$ given by [CFH06] will provide a 3-dimensional model for $\underline{\underline{E}} \tilde{H}$.

Proof of Proposition 5.3. The model constructed is three dimensional, as $\underline{\underline{E}} \widetilde{H}$ and $\mathbb{E}^{2} \times[0,1]$ are both three dimensional; this gives that $\underline{\underline{\operatorname{gd}}}(\Gamma) \leq 3$. Since $\Gamma$ has a subgroup isomorphic to $\mathbb{Z}^{2}$ (consider any $\widetilde{H} \in \mathcal{H}$ ), the result follows from Lemma 2.1

Proposition 5.4. Let $M$ be a closed Seifert fibered manifold with base orbifold modeled on $\mathbb{E}^{2}$, and let $\Gamma=\pi_{1}(M)$ be the fundamental group of $M$. Then

- $M$ is modeled on $\mathbb{E}^{3}$, and $\operatorname{gd}(\Gamma)=4$; or

- $M$ is modeled on $\mathrm{Nil}$, and $\underline{\underline{\underline{\operatorname{gd}}}}(\Gamma)=3$.

Proof. From [Sco83, Theorem 5.3(ii)] we know that $M$ is modeled either on $\mathbb{E}^{3}$ or on Nil. In the former case we have that $\Gamma$ is 3-crystallographic, and by [CFH06] we have that $\operatorname{gd}(\Gamma)=4$.

For the case where $M$ is modeled on Nil, we would like to use [LW12, Theorem 5.13]. For this we will first prove that $\Gamma$ is virtually poly- $\mathbb{Z}$, and then check that $\Gamma$ satisfies $[$ LW12, Theorem 5.13 case $2 \mathrm{~b}]$, so that $\underline{\underline{\operatorname{gd}}}(\Gamma)=3$. The two conditions to check are:

(1) There is an infinite normal subgroup $C \subseteq \Gamma$, and for every infinite cyclic subgroup $D \subseteq \Gamma$ with $\left[\Gamma: N_{\Gamma} D\right]<\infty$ we have $C \cap D \neq 1$.

(2) There exists no subgroup $W \leq \Gamma$ such that its commensurator $N_{\Gamma}[W]$ has virtual cohomological dimension equal to 1 .

From Lemma 3.1 we have the short exact sequence

$$
1 \rightarrow \mathbb{Z} \rightarrow \Gamma \rightarrow \Gamma_{0} \rightarrow 1
$$

where $\Gamma_{0}$ is the orbifold fundamental group of $B$, which is 2-crystallographic by hypotheses, in particular it is virtually poly- $\mathbb{Z}$ with a filtration of the form $1 \subseteq \mathbb{Z} \subseteq \mathbb{Z}^{2} \subseteq \Gamma_{0}$. Since the property of being virtually poly- $\mathbb{Z}$ is closed under taking extensions (see [LW12, Lemma 5.14, i-iv]) we conclude that $\Gamma$ is virtually poly- $\mathbb{Z}$. 
Nil is the continuous Heisenberg Lie group, and can be identified with the group $\mathbb{R}^{3}$ with multiplication given by $(a, b, c) \cdot(d, e, f)=(a+d, b+e, c+f-a e)$. The center of Nil is the subgroup $\{(0,0, z): z \in \mathbb{R}\}$. It is a simple matter to compute conjugates and positive powers:

$$
\begin{aligned}
& (x, y, z)^{-1} \cdot(a, b, c) \cdot(x, y, z)=(a, b, a y-b x+c) \\
& (a, b, c)^{n}=\left(n a, n b, n c-\frac{n(n-1)}{2} a b\right) \text { for } n>0 .
\end{aligned}
$$

We now verify property (1). Let $C \subseteq \Gamma$ be the center $Z(\Gamma)$ of $\Gamma$. We first point out that $C$ is infinite cyclic. Indeed, the group $\Gamma$ can be viewed as a lattice in Nil, which implies $C=\Gamma \cap Z(\mathrm{Nil})$ is a discrete cocompact subgroup in $Z(\mathrm{Nil}) \cong \mathbb{R}$.

Let $D$ be an infinite cyclic subgroup of $\Gamma$, generated by the element $(a, b, c) \in$ Nil. If $(x, y, z) \in$ $N_{\Gamma} D$, then we must have for some $n$ :

$$
(x, y, z)^{-1}(a, b, c)(x, y, z)=(a, b, c)^{n} .
$$

So if $(x, y, z) \in N_{\Gamma} D$ then from the formulas above we see that $n=1$ and $a y-b x=0$.

Without loss of generality suppose $a \neq 0$. Then for $(x, y, z)$ to be a normalizer of $D$, we need $a y-b x=0$, or $y=\frac{b}{a} x$. Thus, we can consider the closed Lie subgroup

$$
H:=\left\{\left(\alpha, \frac{b}{a} \alpha, \beta\right) \mid \alpha, \beta \in \mathbb{R}\right\}
$$

of Nil, and observe that $H$ is isomorphic to $\mathbb{R}^{2}$. Since $N_{\Gamma} D=H \cap \Gamma$, we see that $N_{\Gamma} D$ can be viewed as a discrete subgroup of $H \cong \mathbb{R}^{2}$, which forces $\operatorname{vcd}\left(N_{\Gamma} D\right) \leq 2$. Since $\operatorname{vcd}(\Gamma)=3$, we conclude that $\left[\Gamma: N_{\Gamma} D\right]=\infty$. Thus, for any infinite cyclic $D \leq \Gamma$, either $Z(\Gamma) \cap D \neq\{1\}$ or $\left[\Gamma: N_{\Gamma} D\right]=\infty$; in particular, $\Gamma$ satisfies condition (1) above.

Finally, since $\operatorname{vcd}(\Gamma)=3$, and $N \leq N_{\Gamma} D \leq N_{\Gamma}[D]$ and $\operatorname{vcd}(N)=2$ we verify condition $(2)$ above, completing the proof.

5.2. Seifert fibered manifolds with boundary. In this section we study compact Seifert fibered manifolds with non-empty boundary. Throughout this section, $M$ will always be a compact Seifert fibered manifold with nonempty boundary. Let $\Gamma=\pi_{1}(M)$ be the fundamental group, let $\Gamma_{0}=$ $\pi_{1}^{\text {orb }}(B)$ be the orbifold fundamental group of the base orbifold $B$, and let $\phi: \Gamma \rightarrow \Gamma_{0}$ be the associated homomorphism.

First we will see that we do not have to consider the case of $B$ being a bad orbifold or a good orbifold modeled on $S^{2}$.

Lemma 5.5. Let $M$ be a compact Seifert fibered manifold with nonempty boundary with base orbifold $B$. Denote by $B^{\circ}$ be the interior of $B$. Then $B^{\circ}$ is a good orbifold modeled either on $\mathbb{H}^{2}$ or on $\mathbb{E}^{2}$.

Proof. By the classification of bad orbifolds (see [Sco83, Theorem 2.3]), the only bad orbifolds without boundary have compact underlying space, so $B^{\circ}$ must be good, and therefore finitely covered by a 2-manifold $N^{\circ}$ that is also not compact. Then $N^{\circ}$ is geometric; by the uniformization theorem, all geometric surfaces are modeled on $\mathbb{S}^{2}, \mathbb{E}^{2}$, or $\mathbb{H}^{2}$. Since $\mathbb{S}^{2}$ is compact, all quotients by discrete (finite) actions are also compact, therefore $N^{\circ}$ cannot be modeled on this geometry. The lemma follows.

Proposition 5.6. Let $M$ be a compact Seifert fibered manifold with nonempty boundary. Let $\Gamma=$ $\pi_{1}(M)$, and let $B$ be the base orbifold of $M$. If $B^{\circ}$ is modeled on $\mathbb{E}^{2}$, then $\Gamma$ is 2-crystallographic isomorphic to $\mathbb{Z}^{2}$ or $\mathbb{Z} \rtimes \mathbb{Z}$. In particular $\operatorname{gd}(\Gamma)=3$. 
Proof. Then by Mor05, Theorem 1.2.2] we have that $M$ is modeled on $\mathbb{E}^{3}$ or Nil, or is diffeomorphic to $S^{1} \times S^{1} \times I$, or is a twisted [0,1]-bundle over the Klein bottle. But neither $\mathbb{E}^{3}$ nor Nil admit noncompact geometric quotients. In the remaining two cases the fundamental group of $M$ is isomorphic to $\mathbb{Z}^{2}$ or to $\mathbb{Z} \rtimes \mathbb{Z}$ respectively. Hence $\Gamma$ is a 2-crystallographic group, and the conclusion follows from Lemma 2.1.

Proposition 5.7. Let $M$ be a compact Seifert fiber space with nonempty boundary, and let $\Gamma=$ $\pi_{1}(M)$ be the fundamental group. Suppose that the interior of the base orbifold $B^{\circ}$ is modeled on $\mathbb{H}^{2}$, and has orbifold fundamental group $\pi_{1}^{\text {orb }}(B)=\Gamma_{0}$. Let $\phi: \Gamma \rightarrow \Gamma_{0}$ be the quotient map, and let $\mathcal{A}$ be the collection of preimages of maximal infinite virtually cyclic subgroups of $\Gamma_{0}$. Let $\mathcal{H}$ be a set of representatives of conjugacy classes in $\mathcal{A}$. Consider the following cellular $\Gamma$-push-out:

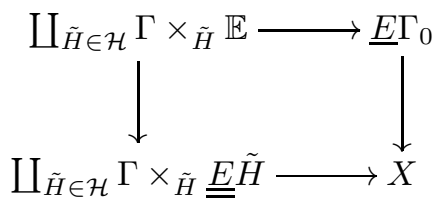

Then $X$ is a model for $\underline{\underline{E}} \Gamma$. In the above cellular $\Gamma$-push-out, we require either (1) the left vertical map is the disjoint union of cellular $\widetilde{H}$-maps $(\widetilde{H} \in \mathcal{H})$, the upper horizontal map is an inclusion of $\Gamma$-CW-complexes, or (2) the left vertical map is the disjoint union of inclusions of $\widetilde{H}$ - $C W$-complexes $(\widetilde{H} \in \mathcal{H})$, the upper horizontal map is a cellular $\Gamma-$ map.

Moreover, $\underline{E} \Gamma_{0}$ admits a 1-dimensional model, and $\underline{\underline{E}} \widetilde{H}$ admits a 3 dimensional model. In particular $\underline{\underline{\operatorname{gd}}}(\Gamma)=3$.

Proof. We would like to use Proposition 4.2 to construct a model for $\underline{\underline{E}}$. Let $\mathcal{F}^{\prime}$ be the family of virtually cyclic subgroups of $\Gamma$ and $\mathcal{F}$ be the family of virtually cyclic subgroups $F$ of $\Gamma$ such that $\phi(F) \leq \Gamma_{0}$ is finite. In order to use Proposition 4.2, we need a model for $E_{\mathcal{F}} \Gamma$, an adapted collection $\mathcal{A}$, and models for $E_{\mathcal{F}} H$ and $\underline{E} H$ for each $H \in \mathcal{A}$.

First, a model for $E_{\mathcal{F}} \Gamma$ is the same as a model for $\underline{E} \Gamma_{0}$. On the other hand $\Gamma_{0}$ contains as a finite index subgroup the fundamental group of a surface with non-empty boundary, therefore $\Gamma_{0}$ is virtually free. Hence $\Gamma_{0}$ admits a splitting as a fundamental grouph of a graph of groups of finite groups, so the Bass-Serre tree $T$ of such a splitting is a model for $\underline{E} \Gamma_{0}$.

Next let us describe an adapted collection $\mathcal{A}$.

Let $\mathcal{A}$ be the collection of subgroups of $\Gamma$ that are preimages of maximal infinite virtually cyclic subgroups of $\Gamma_{0}$. Then we claim that $\mathcal{A}$ is adapted to the pair $\left(\mathcal{F}, \mathcal{F}^{\prime}\right)$ of families of subgroups of $\Gamma$. In fact, the virtually cyclic subgroups of $\Gamma_{0}$ that are conjugate into a vertex group of the graph of groups presentation must be finite, since the vertex groups themselves are finite. In particular, the splitting of $\Gamma_{0}$ given by the graph of groups is acylindrical. By [LO09b, Claim 3], the collection $\mathcal{A}_{0}$ of maximal infinite virtually cyclic subgroups of $\Gamma_{0}$ is adapted to the pair $\left(\mathcal{F} \mathcal{I N} \mathcal{N}_{0}, \mathcal{V} \mathcal{C}_{0}\right)$ of families of finite and virtually cyclic subgroups of $\Gamma_{0}$, respectively. By Lemma $4.4, \mathcal{A}=\widetilde{\mathcal{A}_{0}}$ is therefore adapted to the pair $(\mathcal{F}, \widetilde{\mathcal{V C}})$ of families of subgroups of $\Gamma$. Since $\mathcal{F} \subseteq \mathcal{F}^{\prime} \subseteq \widetilde{\mathcal{V C}_{0}}$, Lemma 4.3 shows that $\mathcal{A}$ is adapted to the pair $\left(\mathcal{F}, \mathcal{F}^{\prime}\right)$, as claimed.

Let $\widetilde{H} \in \mathcal{A}$ be the $\phi$-preimage of a maximal infinite virtually cyclic subgroup $H \leq \Gamma_{0}$. A model for $E_{\mathcal{F}} \widetilde{H}$ is the same as a model for $\underline{E} H$. Since $H$ is virtually cyclic, $\mathbb{E}$ is a model for $\underline{E} H$.

It remains to construct a model for $\underline{\underline{E}} \widetilde{H}$. But this can be done by an argument identical to the one at the end of Proposition 5.2 We leave the details to the reader. 
Remark 5.8. Note that in both cases whether the base orbifold is modeled on $\mathbb{E}^{2}$ or $\mathbb{H}^{2}$ we have explicit models for $\underline{\underline{E}} G$. In fact, in Proposition [5.6] we can use the construction of [CFH06]. While in Proposition 5.7 we have an explicit push-out where $\underline{E} \Gamma_{0}$ is a tree and every $\tilde{H}$ has finite normal subgroup such that the quotient is crystallographic, and again we can use CFH06.

Remark 5.9. Note that the adapted collection $\mathcal{A}$ constructed in the proof of Proposition 5.7. consists of preimages of maximal infinite virtually cyclic subgroups of $\Gamma_{0}$ in $\Gamma$. So $\mathcal{H}$ contains representatives of the conjugacy classes of the boundary torus of $M$. This fact will be used in some of the Bredon cohomology computations in Section 9 ,

\section{The HYPERBolic CASE}

In this section, we will analyze the geometric dimension of lattices in the isometry group $\operatorname{PSL}(2, \mathbb{C})$ of hyperbolic 3-space. Since we are going to use some standard properties of hyperbolic 3-dimensional geometry we refer the reader to [Sco83, p. 448] for details about the geometry of $\mathbb{H}^{3}$.

Proposition 6.1. Let $M$ be a connected, oriented, finite-volume hyperbolic 3-manifold, and let

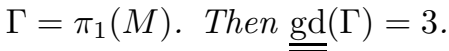

Proof. In order to establish the proposition, we start by using a push-out construction to create a model for $\underline{\underline{E}} \Gamma$. This will provide an upper bound on $\underline{\underline{\operatorname{dg}}}(\Gamma)$.

The group $\Gamma$ is a relatively hyperbolic group, relative to the collection of maximal parabolic subgroups $P_{\xi}$ of $\Gamma$. From [LO07, Theorem 2.6], we know that the collection $\mathcal{A}$ of infinite maximal subgroups $M_{c}$ that stabilize a geodesic $c(\mathbb{R}) \subset \mathbb{H}^{3}$ and infinite maximal parabolic subgroups $P_{\xi}$ that fix a unique boundary point $\xi \in \partial \mathbb{H}^{3}$ is adapted to the pair $\left(F_{I N}, V_{C Y C}\right)$.

Let $\mathcal{A}$ be the collection of infinite maximal $M_{c}$ or $P_{\xi}$ subgroups of $\Gamma$. Let $\mathcal{H}$ be a complete set of representatives of the conjugacy classes within $\mathcal{A}$, and consider the following cellular $\Gamma$-push-out:

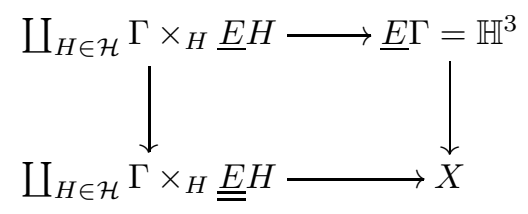

Then Proposition 4.2 tells us that $X$ is a model for $\underline{E} \Gamma$. Since $X$ is 3-dimensional, we obtain the inequality $\operatorname{gd}(\Gamma) \leq 3$. If $\Gamma$ is nonuniform, it contains subgroups isomorphic to $\mathbb{Z}^{2}$, and the conclusion follows from Lemma 2.1 .

Suppose now that $\Gamma$ is a uniform lattice. The push-out construction above gives rise to the Mayer-Vietoris sequence

$$
\cdots \rightarrow \underline{\underline{H}}^{3}(\Gamma ; \underline{\mathbb{Z}}) \rightarrow\left(\bigoplus_{H \in \mathcal{H}} \underline{\underline{H}}^{3}(H ; \underline{\mathbb{Z}})\right) \oplus \underline{\underline{H}}^{3}(\Gamma ; \underline{\mathbb{Z}}) \rightarrow \bigoplus_{H \in \mathcal{H}} \underline{\underline{H}}^{2}(H ; \underline{\mathbb{Z}}) \rightarrow \cdots
$$

Note that in this case $H$ is always of the form $M_{c}$ (there are no $P_{\xi}$ elements in $\mathcal{H}$ because we have no parabolic elements), hence it is virtually cyclic. Moreover, we have $\underline{H}^{3}(\Gamma ; \underline{\mathbb{Z}}) \cong H^{3}(M ; \mathbb{Z}) \cong \mathbb{Z}$. The Mayer-Vietoris sequence thus simplifies to

$$
\cdots \rightarrow \underline{\underline{H}}^{3}(\Gamma ; \underline{\mathbb{Z}}) \rightarrow \mathbb{Z} \rightarrow 0 \rightarrow \ldots
$$

This gives the lower bound $3 \leq \underline{\underline{\mathrm{cd}}}(\Gamma) \leq \underline{\underline{\operatorname{gd}}}(\Gamma)$ and completes the proof. 


\section{Two EXCEPTIONAL CASES}

In this section, we focus on manifolds whose JSJ decomposition has all pieces that are Seifert fibered with Euclidean base orbifold. We let $K$ denote the twisted I-bundle over the Klein bottle. Note that, while the Klein bottle is a non-orientable surface, the space $K$ is an orientable 3-manifold with a torus boundary.

Lemma 7.1. Let $M$ be an irreducible 3-manifold, and assume that all the pieces in the JSJ decomposition are Seifert fibered with Euclidean base orbifold. Then either:

(1) $M$ is a torus bundle over $S^{1}$, or

(2) $M$ consists of two copies of $K$ glued together along their boundary.

Proof. From Proposition 5.6 we know the only such Seifert fibered pieces are either (i) the torus times an interval, and (ii) the twisted $I$-bundle $K$ over the Klein bottle. If we have a piece of type (i) whose boundary tori are distinct in $M$, then we would violate the minimality of the number of tori in the JSJ decomposition. So if we have a piece of type (i), then the JSJ decomposition of $M$ in fact has a single piece, and $M$ must be a torus bundle over $S^{1}$. If there are no pieces of type (i), then the decomposition of $M$ consists of two copies of $K$ identified together.

We will now compute the virtually cyclic geometric dimension for these classes of manifolds.

\subsection{Torus bundles over the circle.}

Proposition 7.2. Let $M$ be a torus bundle over $S^{1}$, with fundamental group $\Gamma$. Then exactly one of the following happens:

(1) $M$ is modeled on $\mathbb{E}^{3}$, hence $\operatorname{gd}(\Gamma)=4$,

(2) $M$ is modeled on $\mathrm{Nil}$, and $\mathrm{g} \overline{\overline{\mathrm{d}}(\Gamma)}=3$,

(3) $M$ is modeled on Sol, and $\overline{\underline{\underline{\operatorname{gd}}}}(\Gamma)=3$.

Proof. Since $M$ is a torus bundle over $S^{1}, \Gamma \cong \mathbb{Z}^{2} \rtimes_{\varphi} \mathbb{Z}$ with $\varphi: \mathbb{Z} \rightarrow S L_{2}(\mathbb{Z})$. Denote $\varphi(1)=A$. We have three cases depending on whether the matrix $A$ is elliptic, parabolic or hyperbolic (these cases correspond to whether the trace of $A$ is $<2,=2$ and $>2$ respectively).

If $A$ is elliptic it has finite order, so $\Gamma$ is virtually $\mathbb{Z}^{3}$. This implies $M$ is finitely covered by

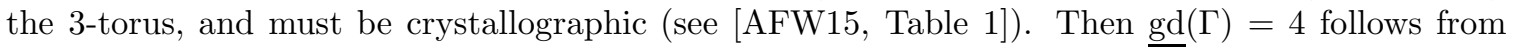
CFH06.

If $A$ is parabolic, then the action on $\mathbb{Z}^{2}$ has an invariant rank one subgroup. This implies that the center $Z$ of $\Gamma^{\prime}$ is infinite cyclic. Since $Z$ is a characteristic group of $\Gamma^{\prime}$, it follows that $Z$ is an infinite cyclic normal subgroup of $\Gamma$. Applying [Por08, Theorem 7], we see that $M$ is Seifert fibered with virtually nilpotent (but not virtually abelian) fundamental group, so is modeled on Nil (see

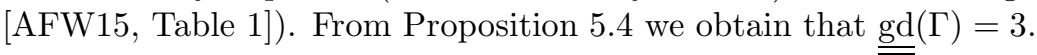

Finally, consider the case where $A$ is hyperbolic. Then the action of $A$ on $\mathbb{Z}^{2}$ does not have any non-trivial invariant subgroups. This implies the center of $\Gamma$ is trivial, so by [Thu97, Theorem 4.7.13] we obtain that $M$ is modeled on Sol. In order to compute $\operatorname{gd}(\Gamma)$, we will verify that every finite index subgroup of $\Gamma$ has finite center. Let $H \leq \Gamma$ be a finite index subgroup. Then we have the short exact sequence

$$
1 \rightarrow H \cap \mathbb{Z}^{2} \rightarrow H \rightarrow \mathbb{Z} \rightarrow 1 .
$$

Then $H \cap \mathbb{Z}^{2}$ has finite index in $\mathbb{Z}^{2}$, and $p(H) \neq 0$. $A$ is hyperbolic, so every positive power of $A$ is also hyperbolic. This again implies that the centralizer $Z_{\Gamma}(H)$ must be trivial. Recalling that $H$ was an arbitrary finite index subgroup of $\Gamma,[\mathrm{LW12}$, Theorem 5.13] allows us to conclude $\underline{\underline{\operatorname{gd}}}(\Gamma)=3$. 


\subsection{Twisted doubles of $K$.}

Proposition 7.3. Let $M$ be an irreducible 3-manifold obtained as the union of two copies of $K$, where the gluing is via a homeomorphism $\varphi: T \rightarrow T$ between the boundary torus. Denote by $\Gamma$ the fundamental group of $M$. Then exactly one of the following happens:

(1) $M$ is modeled on $\mathbb{E}^{3}$, hence $\operatorname{gd}(\Gamma)=4$,

(2) $M$ is modeled on $\mathrm{Nil}$, and $\mathrm{g} \overline{\overline{\mathrm{d}}(\Gamma)}=3$,

(3) $M$ is modeled on Sol, and $\underline{\underline{\underline{\operatorname{gd}}}}(\Gamma)=3$.

Proof. We know that $\pi_{1}(K)$ is isomorphic to the fundamental group of the Klein bottle $\mathbb{Z} \rtimes \mathbb{Z}$. This implies $\Gamma \cong(\mathbb{Z} \rtimes \mathbb{Z}) *_{\mathbb{Z}^{2}}(\mathbb{Z} \rtimes \mathbb{Z})$, where the $\mathbb{Z}^{2}$, which embeds as an index two subgroup of $\mathbb{Z} \rtimes \mathbb{Z}$, comes from the boundary of $K$. Note that the $\mathbb{Z}^{2}$ subgroup is a normal subgroup of $\Gamma$, so can be identified with the kernel of an induced surjective morphism $p: \Gamma \rightarrow D_{\infty}$. Defining $\Gamma^{\prime}:=p^{-1}(\mathbb{Z})$ to be the pre-image of the cyclic index two subgroup of the infinite dihedral group $D_{\infty}$, we obtain the diagram

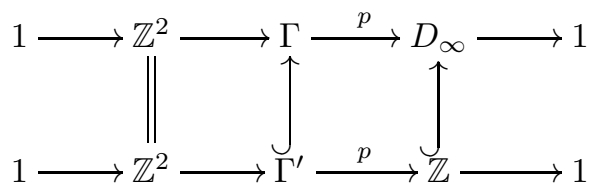

Thus we see that $\Gamma^{\prime}$ is one of the groups discussed in Proposition 7.2. Since $\Gamma$ contains $\Gamma^{\prime}$ as an index two subgroup, we see that the geometry of $M$ coincides with the geometry of the corresponding double cover $M^{\prime}$ (see the algebraic criteria in AFW15, Table 1]).

The calculations of $\operatorname{gd}(\Gamma)$ then follow from [CFH06] in the $\mathbb{E}^{3}$ case, and from Proposition $[5.4$ in the Nil case. In the Sol case, just as in Proposition [7.2, one can easily verify that $\Gamma$ satisfies the conditions of [LW12, Theorem 5.13], which gives us $\underline{\underline{\operatorname{gd}}}(\Gamma)=3$ (the details are left to the reader).

Let us summarize the information we have so far on the JSJ decomposition of $M$, when $M$ is not geometric.

Corollary 7.4. Let $M$ be a prime 3-manifold, which we assume is not geometric, and let $N_{i}$ be the pieces in the JSJ decomposition of $M$. Then all the $N_{i}$ are either (i) hyperbolic, (ii) Seifert fibered over a hyperbolic base, or (iii) copies of K, the twisted I-bundle over the Klein bottle.

Moreover, every piece of type (iii) is attached to a piece of type (i) or (ii). In particular, there must be a piece of type (i) or (ii).

Proof. There must be at least one torus in the decomposition, for otherwise $M$ itself is closed hyperbolic or closed Seifert fibered, hence geometric. By Lemma 7.1 and Proposition 7.2, there are no pieces homeomorphic to $T^{2} \times[0,1]$, so the only pieces that are Seifert fibered over a Euclidean base 2-orbifold are copies of $K$. Finally, if a piece of type (iii) is attached to a piece of type (iii), then Proposition 7.3 tells us $M$ is geometric.

\section{Reducing to the JSJ PIECES}

Next we relate the study of the virtually cyclic geometric dimension of the fundamental group of a prime manifold, to that of the components in its JSJ decomposition. In Sections 5 and 6 , we have already calculated the virtually cyclic geometric dimension of the prime manifolds that are geometric. So throughout this section, we will work exclusively with non-geometric prime 3 -manifolds. 
Theorem 8.1. Let $M$ be a closed, oriented, connected, prime 3-manifold which is not geometric. Let $N_{1}, \ldots, N_{k}$ with $k \geq 1$, be the components arising in the JSJ decomposition. Denote $G=\pi_{1}(M)$, $G_{i}=\pi_{1}\left(N_{i}\right)$. Let $X_{i}$ be an arbitrary model for $\underline{\underline{E}} G_{i}$. Then

$$
3 \leq \max \left\{\underline{\underline{\operatorname{gd}}}\left(G_{i}\right) \mid 1 \leq i \leq k\right\} \leq \underline{\underline{\operatorname{gd}}}(G) \leq \max \left\{4, \operatorname{dim}\left(X_{i}\right) \mid 1 \leq i \leq k\right\} .
$$

Proof. Since $M$ is not geometric, it has at least one torus in its JSJ decomposition (see Corollary 7.4), so we will have a subgroup of $G$ isomorphic to $\mathbb{Z}^{2}$. Moreover, every $G_{i}$ has a subgroup isomorphic to $\mathbb{Z}^{2}$, giving us the first inequality. The second inequality follows from Lemma 2.1. For the last inequality, we proceed as in the proof of Theorem 4.11. The JSJ decomposition provides a splitting of $G$ as the fundamental group of a graph of groups with vertex groups $G_{i}$ and edge groups copies of $\mathbb{Z}^{2}$, and by Lemma 2.1 we have $\operatorname{gd}\left(\mathbb{Z}^{2}\right)=3$. Now the conclusion will follow from Corollary 4.10 once we prove that the splitting of $\overline{\bar{G}}$ is acylindrical, which is done below in Proposition 8.2

Proposition 8.2. Let $M$ be a closed, oriented, connected, prime 3-manifold, which is not geometric. Let $\mathbf{Y}$ be the graph of groups associated to its JSJ decomposition. Then the splitting of $G=\pi_{1}(M)$ as the fundamental group of $\mathbf{Y}$ is acylindrical.

Proof. Let $T$ be the Bass-Serre covering tree of $\mathbf{Y}$, and let $c$ be a path of length 5 . $G$ acts without inversion on $T$, so elements that stabilize $c$ must in fact fix it, for otherwise they would invert the center edge of $c$. We will argue that the stabilizer of $c$ is trivial. This will show that the splitting satisfies the definition of acylindricity, with integer $k=5$.

Let $c$ have edges $\left\{\widetilde{y}_{1}, \ldots, \widetilde{y}_{5}\right\}$, and let $o\left(\widetilde{y}_{i}\right)=\widetilde{P}_{i-1}, t\left(\widetilde{y}_{i}\right)=\widetilde{P}_{i}$ in $T$. Let $y_{i}=p\left(\widetilde{y}_{i}\right) \in \operatorname{edge} Y$ and $P_{i}=p\left(\widetilde{P}_{i}\right) \in \operatorname{vert} Y$ for each vertex and edge in $c$. Let $N_{i}, 0 \leq i \leq 5$, be the manifolds that correspond to each vertex $P_{i}$, with fundamental groups $G_{i}$. Let $T_{i}, 1 \leq i \leq 5$, be the torus associated to each edge $y_{i}$, and denote by $Z_{i}$ the stabilizer of $\tilde{y}_{i}$ (which is a conjugate in $G$ of the fundamental group of $T_{i}$ ).

Now suppose that one of the $N_{i}(1 \leq i \leq 4)$ is hyperbolic. Then the stabilizer of $c$ is contained in $Z_{i-1} \cap Z_{i}$. The groups $Z_{i-1}$ and $Z_{i}$ are stabilizers of two distinct points in the boundary at infinity of $\mathbb{H}^{3}$. It follows that the group that fixes $c$ must be finite, hence trivial since the $G_{i}$ is torsion-free.

So we now need to consider the case where all the $N_{i}(1 \leq i \leq 4)$ are Seifert fibered. Recall that the $N_{i}$ have non-empty boundary, so there are only two possible cases for each of their base orbifold: either the base is hyperbolic, or it is Euclidean. In view of Corollary 7.4) either $N_{2}$ or $N_{3}$ is Seifert fibered with hyperbolic base 2-orbifold.

Let us now briefly pause and focus on $N$ a Seifert fibered space, with hyperbolic base 2-orbifold. Then by [Mor05, Theorem 1.2.2], $G=\pi_{1}(N)$ acts on $\mathbb{H}^{2} \times \mathbb{R}$, with quotient the corresponding $N_{i}$. Notice an important feature of such Seifert fibered spaces - they come equipped with a canonical Seifert fibered structure. Indeed, the circle fibers in $N_{i}$ always lift to copies of the $\mathbb{R}$ factor in the universal cover.

Each edge incident to the corresponding vertex $\tilde{P}$ has stabilizer a $\mathbb{Z}^{2}$ subgroup of $G$. Up to reparametrization, the $\mathbb{Z}^{2}$-action on the universal cover $\mathbb{H}^{2} \times \mathbb{R}$ is described as follows. The first coordinate acts by translation in the $\mathbb{R}$-factor, while the second coordinate acts by a parabolic isometry on the $\mathbb{H}^{2}$-factor. Noting that a pair of parabolic isometries that are centered at different points at infinity always intersect trivially, we conclude that the corresponding pair of edge stabilizers can only intersect in an infinite cyclic subgroup. Moreover, the axes of translation of this cyclic subgroup corresponds precisely to the fibers of the Seifert fibration on $N$.

We now continue our proof. If both $N_{2}, N_{3}$ are Seifert fibered with hyperbolic base, then we claim that $Z_{1} \cap Z_{2} \cap Z_{3}$ is trivial. By the discussion above, $Z_{1} \cap Z_{2}$ is an infinite cyclic subgroup 
of $G_{2}$, generated by the Seifert fibers of $N_{2}$. Similarly, $Z_{2} \cap Z_{3}$ is also an infinite cyclic subgroup of $G_{3}$, generated by the Seifert fibers of $N_{3}$. Consider the torus $T_{2}$ (with fundamental group $Z_{2}$ ) where $N_{2}$ and $N_{3}$ are glued together. This 2-torus has two circle fibrations induced on it, depending on whether we view it as a subspace of $N_{2}$ or of $N_{3}$. The circle fibers induced by the $N_{2}$ fibration correspond to the subgroup $Z_{1} \cap Z_{2}$, while the circle fibers induced by the $N_{3}$ fibration correspond to the subgroup $Z_{2} \cap Z_{3}$. If these two subgroups intersect non-trivially, then the two fibrations match on the common 2-torus $T_{2}$, and we obtain a Seifert fibered structure on $N_{2} \cup_{T_{2}} N_{3}$. But this contradicts the minimality of the JSJ decomposition. Thus the two fibrations on $T_{2}$ cannot match, and hence $\left(Z_{1} \cap Z_{2}\right) \cap\left(Z_{2} \cap Z_{3}\right)$ is trivial. But this group is precisely the intersection of the stabilizers of the three consecutive edges $\tilde{y}_{1}, \tilde{y}_{2}, \tilde{y}_{3}$ in the path $c$. Since this intersection contains the stabilizer of $c$, we conclude that $c$ has trivial stabilizer.

Finally, we are left with one remaining case: one of $N_{2}, N_{3}$ is Seifert fibered with hyperbolic base, while the other one is Seifert fibered with flat base. Without loss of generality, we assume that $N_{2}$ has hyperbolic base, while $N_{3}$ has flat base. Then as discussed above, we see that $N_{3}$ must coincide with $K$, the twisted interval bundle over the Klein bottle. In particular, $G_{3}=\pi_{1}\left(N_{3}\right)$ coincides with the fundamental group of the Klein bottle, and the single boundary torus $\partial N_{3}$ has fundamental group $Z_{2}=Z_{3}$ which is the canonical index two $\mathbb{Z}^{2}$ subgroup in $G_{3}$.

Let us briefly focus on the manifold $K$. The universal cover of $K$ is $\mathbb{R}^{2} \times[0,1]$. There are precisely two possible Seifert fibrations of $K$. Indeed, a Seifert fibration lifts to a foliation of the universal cover $\mathbb{R}^{2} \times[0,1]$ by parallel straight lines. In order to descend to a well-defined foliation of $K$, the straight lines have to be invariant under the action of the $\pi_{1}(K)$, the fundamental group of the Klein bottle. Since $\pi_{1}(K)$ is crystallographic, we have a well-defined holonomy involution $h: \mathbb{Z}^{2} \rightarrow \mathbb{Z}^{2}$, given by conjugating the normal index two subgroup $\mathbb{Z}^{2} \triangleleft \pi_{1}(K)$ by any element in $\pi_{1}(K) \backslash \mathbb{Z}^{2}$. This holonomy action leaves invariant precisely two cyclic subgroups of $\mathbb{Z}^{2}$, corresponding to the \pm 1 eigenspaces of $h$. The foliations with slopes matching the eigenspace of $h$ are precisely the ones which will descend to $K$.

Continuing our proof, the canonical Seifert fibered structure on the piece $N_{2}$ induces foliations by straight lines on $\mathbb{R}^{2} \times\{0\} \subset \tilde{N}_{3}$. It also induces a foliation by straight lines on $\mathbb{R}^{2} \times\{1\} \subset \tilde{N}_{3}$. These two foliations are related: the foliation on $\mathbb{R}^{2} \times\{1\}$ can be obtained from the foliation on $\mathbb{R}^{2} \times\{0\}$, by applying the holonomy map $h$. There are now two possible cases: either these foliations have slope matching an eigenspace of $h$, or they will not.

If the foliation matches the eigenspace of $h$, then putting the corresponding Seifert structure on $N_{3}$, we obtain a globally defined Seifert structure on $N_{2} \cup_{T_{2}} N_{3}$. This contradicts the minimality condition in the JSJ splitting.

On the other hand, if the slope does not match an eigenspace, then from the action of the holonomy $h$, we see that the straight line foliations on the two sides $\mathbb{R}^{2} \times\{0\}$ and $\mathbb{R}^{2} \times\{1\}$ are by lines of different slope. But this means that, if we view the infinite cyclic groups $Z_{1} \cap Z_{2}$ and $Z_{3} \cap Z_{4}$ as subgroups of $\mathbb{Z}^{2} \triangleleft \pi_{1}(K)=G_{3}$, they act by translations in distinct directions, and hence the intersection $\left(Z_{1} \cap Z_{2}\right) \cap\left(Z_{3} \cap Z_{4}\right)$ is trivial. Since the stabilizer of $c$ is contained within this intersection, it is also trivial. This was the last remaining case, and hence completes the proof that the splitting is acylindrical.

\section{BREDON COHOMOLOGY COMPUTATION}

From our work in Sections 5 and 6 , we know the geometric dimension for fundamental groups of closed geometric 3-manifolds. In this section, we focus on non-geometric prime 3-manifolds. 
Proposition 9.1. Let $M$ be a closed oriented prime 3-manifold, with $\Gamma=\pi_{1}(M)$, and assume that $M$ is not geometric. Then $\underline{\underline{\operatorname{gd}}}(\Gamma)=3$.

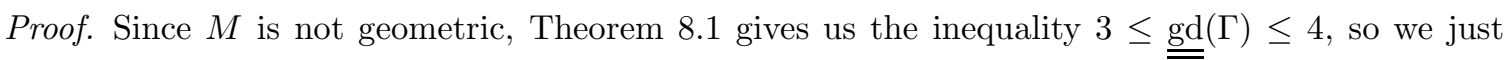
need to rule out $\underline{\operatorname{gd}}(\Gamma)=4$.

Let $\mathbf{Y}$ be the graph of groups associated to the JSJ decomposition of $M$, so that $\pi_{1}(\mathbf{Y})=\pi_{1}(M)=$ $\Gamma$. Then the $G_{i}$ are the groups associated to the vertices $p_{i} \in$ vert $Y$. Proposition 8.2 tells us that $\mathbf{Y}$ is an acylindrical graph of groups. Letting $\mathcal{F}$ be the family of virtually cyclic subgroups of $\Gamma$ that are conjugate into one of the $G_{i}$, Proposition 4.9 tells us that an $\underline{\underline{E}} \Gamma$ can be obtained by attaching 2-cells to a model for $E_{\mathcal{F}} \Gamma$. Thus our proposition would follow immediately from the

Claim: There exists a 3-dimensional model for $E_{\mathcal{F}} \Gamma$.

Unfortunately the naïve model for $E_{\mathcal{F}} \Gamma$ described in the proof of Proposition 4.7 is 4-dimensional. In order to show that there exists a 3-dimensional model, we will instead show that the fourth Bredon cohomology $H_{\mathcal{F}}^{4}(\Gamma ; F)$ vanishes for all coefficient modules $F \in \operatorname{Mod}-\mathcal{O}_{\mathcal{F}} \Gamma$. This implies that the Bredon cohomological dimension $\operatorname{cd}_{\mathcal{F}} \Gamma=3$, which implies the existence of the desired 3-dimensional model (see Lemma 2.1).

To show $H_{\mathcal{F}}^{4}(\Gamma ; F)=0$, we make use of the graph of spaces model described in Proposition 4.7 Chose an orientation $A$ of the edges of the finite graph Y. Then by [MP02, Remark 4.2], the graph of spaces gives rise to the long exact sequence

$$
\ldots \stackrel{\partial^{*}}{\rightarrow} \bigoplus_{\text {vert } Y} \underline{\underline{H}}^{3}\left(G_{i} ; F\right) \stackrel{\alpha^{*}}{\rightarrow} \bigoplus_{y \in A} \underline{\underline{H}}^{3}\left(\mathbb{Z}^{2} ; F\right) \stackrel{\iota^{*}}{\rightarrow} H_{\mathcal{F}}^{4}(\Gamma ; F) \stackrel{\partial^{*}}{\rightarrow} \bigoplus_{\text {vert } Y} \underline{\underline{H}}^{4}\left(G_{i} ; F\right) \rightarrow \ldots
$$

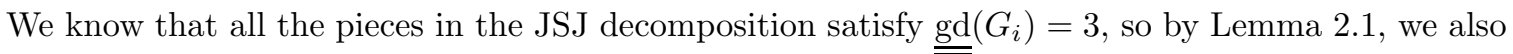
have $\underline{\underline{\mathrm{cd}}}\left(G_{i}\right)=3$. This forces $\underline{\underline{H}}^{4}\left(G_{i} ; F\right)=0$ for all the $G_{i}$. Thus in order to prove that $H_{\mathcal{F}}^{4}(\Gamma ; F)$ is trivial, it suffices to prove that

$$
\alpha^{*}: \bigoplus_{\text {vert } Y} \underline{\underline{H}}^{3}\left(G_{i} ; F\right) \rightarrow \bigoplus_{y \in A} \underline{\underline{H}}^{3}\left(\mathbb{Z}^{2} ; F\right)
$$

is surjective. Given an oriented edge $y \in A$, and one of the endpoints $p_{i} \in \operatorname{vert} Y$, the corresponding morphism $\underline{\underline{H}}^{3}\left(G_{i} ; F\right) \rightarrow \underline{\underline{H}}^{3}\left(\mathbb{Z}^{2} ; F\right)$ is induced by the inclusion $\mathbb{Z}^{2} \hookrightarrow G_{i}$, corresponding to one of the boundary tori $T^{2} \hookrightarrow N_{i}$. We know from Corollary 7.4 that each piece $N_{i}$ from the JSJ decomposition has nonempty boundary and is either (i) hyperbolic, (ii) Seifert fibered with hyperbolic base 2orbifold, or (iii) a copy of $K$, the twisted $I$-bundle over the Klein bottle. Let us analyze the morphism in the first two cases.

In case (i), $N_{i}$ is hyperbolic with non-empty boundary and fundamental group $G_{i}$. Then Proposition 6.1 gives the following Mayer-Vietoris exact sequence

$$
\ldots \rightarrow \underline{\underline{H}}^{3}\left(G_{i} ; F\right) \rightarrow\left(\bigoplus_{H \in \mathcal{H}} \underline{\underline{H}}^{3}(H ; F)\right) \oplus \underline{\mathrm{H}}^{3}\left(G_{i} ; F\right) \rightarrow \bigoplus_{H \in \mathcal{H}} \underline{\mathrm{H}}^{3}(H ; F) \rightarrow \ldots
$$

Since each $H \in \mathcal{H}$ is either 2-crystallographic or virtually cyclic, we always have that $\underline{H}^{3}(H ; F)=0$. Also, among the elements of $\mathcal{H}$ we have the fundamental groups of the boundary tori of $G_{i}$, lets call $\mathcal{H}^{\prime} \subset \mathcal{H}$ the subset consisting of those copies of $\mathbb{Z}^{2}$. Then for every $\mathcal{H}^{\prime \prime} \subseteq \mathcal{H}^{\prime}$ we obtain from the Mayer-Vietoris above that the map

$$
\underline{\underline{\mathrm{H}}}^{3}\left(G_{i} ; F\right) \rightarrow \bigoplus_{H \in \mathcal{H}^{\prime \prime}} \underline{\underline{\mathrm{H}}}^{3}(H ; F)
$$




\begin{tabular}{|l|c|l|}
\hline Type of geometric piece & Analyzed in & $\underline{\underline{g d}(\Gamma)}$ \\
\hline \hline $\begin{array}{l}\text { Compact Seifert fibered piece with bad base orbifold } \\
\text { or good base orbifold modeled on } S^{2}\end{array}$ & Impossible, Lemma 5.5 & - \\
\hline $\begin{array}{l}\text { Compact Seifert fibered piece with base orbifold } \\
\text { modeled on } \mathbb{H}^{2}\end{array}$ & Proposition [5.7 & 3 \\
\hline $\begin{array}{l}\text { Compact Seifert fibered piece with base orbifold } \\
\text { modeled on } \mathbb{E}^{2}\end{array}$ & Proposition [5.6 & 3 \\
\hline Hyperbolic piece & Proposition [6.1 & 3 \\
\hline
\end{tabular}

TABLE 1. Virtually cyclic dimension of pieces in the JSJ decomposition.

\begin{tabular}{|l|l|l|l|}
\hline Type of closed 3-manifold & Analyzed in & gd $(\Gamma)$ & Geometry \\
\hline \hline $\begin{array}{l}\text { Seifert fibered with bad base orbifold or good base } \\
\text { orbifold modeled on } S^{2}\end{array}$ & Proposition 5.1 & 0 & $\begin{array}{l}S^{3} \\
S^{2} \times \mathbb{E}\end{array}$ \\
\hline $\begin{array}{l}\text { Seifert fibered manifold with base orbifold modeled } \\
\text { on } \mathbb{H}^{2}\end{array}$ & Proposition [5.3 & 3 & $\begin{array}{l}\mathbb{H}^{2} \\
\mathrm{PSL}_{2}(\mathbb{R})\end{array}$ \\
\hline $\begin{array}{l}\text { Seifert fibered manifold with base orbifold modeled } \\
\text { on } \mathbb{E}^{2}\end{array}$ & Proposition $[5.4$ & 4 or 3 & $\begin{array}{l}\mathbb{E}^{3} \text { or Nil } \\
\text { resp. }\end{array}$ \\
\hline Hyperbolic manifold & Proposition 6.1 & 3 & $\mathbb{H}^{3}$ \\
\hline
\end{tabular}

TABLE 2. Virtually cyclic dimension of closed geometric manifolds.

induced by the inclusion of subgroups is surjective.

Next, let us analyze case (ii), where $N_{i}$ is Seifert fibered with $B$ modeled on $\mathbb{H}^{2}$ and fundamental group $G_{i}$. Then using the push-out from Proposition 5.7, an argument similar to the one in the hyperbolic case, shows that the map

$$
\underline{\underline{\mathrm{H}}}^{3}\left(G_{i} ; F\right) \rightarrow \bigoplus_{H \in \mathcal{H}^{\prime \prime}} \underline{\underline{\mathrm{H}}}^{3}(H ; F)
$$

is again surjective for every subset $\mathcal{H}^{\prime \prime} \subset \mathcal{H}$, where again $\mathcal{H}$ is the set of $\mathbb{Z}^{2}$ subgroups in $G_{i}$ corresponding to boundary components of $N_{i}$.

Note that in case (iii), where $N_{i}$ is the twisted interval bundle over the Klein bottle, it is not clear how to prove a surjectivity statement as above, as we do not have a push-out construction for the corresponding classifying space.

We now return to the proof of the Proposition. We needed to show that the morphism $\alpha^{*}$ in Equation (2) is surjective. The only possible difficulty lies from the $\mathbb{Z}^{2}$ subgroups that arise as boundaries of geometric pieces homeomorphic to $K$ (see last paragraph). But from Corollary 7.4 . every geometric piece $N_{i}$ that is homeomorphic to $K$ gets attached to another geometric piece $N_{j}$ that is not homeomorphic to $K$. In particular, the corresponding morphism $\underline{\underline{H}}^{3}\left(G_{j} ; F\right) \rightarrow \underline{\underline{H}}^{3}(H ; F)$ is surjective (where $H \cong \mathbb{Z}^{2}$ is the subgroup corresponding to the 2-torus $\overline{\bar{\partial}} N_{i}$ ). It is now easy to see that the morphism in Equation (2) is in fact surjective, completing our proof.

\section{Proof of the MAIN THEOREM}

We are now ready to establish our main theorem. 
Proof of Theorem 1.1. First, we verify that $\operatorname{gd}(\Gamma) \leq 4$ for every closed orientable 3 -manifold $M$. In view of Theorem 4.11, it is sufficient to consider the case where $N$ is prime. We have two cases depending on whether $N$ is geometric or not. If $N$ is a closed geometric 3-manifold, then $\operatorname{gd}(\Gamma) \leq 4$ always holds - see Table 2 for details. On the other hand, if $N$ is a prime 3-manifold which is not geometric, then Proposition 9.1 shows that $\operatorname{gd}(\Gamma)=3$.

Having established that $\operatorname{gd}(\Gamma) \leq 4$ for all closed orientable 3-manifolds, let us now analyze the possibilities for $\operatorname{gd}(\Gamma)$, and establish statements (1)-(4) in our main theorem.

Statement (1). For every group $\Gamma$ and every family $\mathcal{F}$ of subgroups we have that $\operatorname{gd}_{\mathcal{F}}(\Gamma)=0$ if and only if $\Gamma \in \mathcal{F}$. Statement (1) follows as a particular case.

Statement (2). Assume that $\operatorname{gd}(\Gamma)=2$. Then it follows from Theorem 4.11that all the components in the prime decomposition have virtually cyclic geometric dimension at most 2. Proposition 9.1 then tells us that all the prime factors of $M$ are geometric. Looking at Table 2, we see all the components in the prime decomposition must be modeled on $S^{2} \times \mathbb{E}$ or $S^{3}$, and hence have virtually cyclic fundamental group. Thus $\Gamma$ is a free product of virtually cyclic groups.

Conversely, if $\Gamma$ is a free product of virtually cyclic groups, then we have an acylindrical splitting of $\Gamma$. By Corollary 4.10 we obtain $\operatorname{gd}(\Gamma) \leq 2$. To obtain a lower bound we just have to observe that $\Gamma$ always contains a free group on $\overline{\overline{t w o}}$ generators. Since such groups have virtually cyclic dimension equal to 2 , we obtain $2 \leq \underline{\underline{\operatorname{gd}}}(\Gamma)$.

Statement (3). If $\Gamma$ contains a $\mathbb{Z}^{3}$ subgroup, applying Lemma 2.1 gives the lower bound $4=$ $\underline{\underline{\operatorname{gd}}}\left(\mathbb{Z}^{3}\right) \leq \underline{\underline{\operatorname{gd}}}(\Gamma)$, which forces $\underline{\underline{\operatorname{gd}}}(\Gamma)=4$. Conversely, in view of Theorem 4.11, if $\underline{\operatorname{gd}}(\Gamma)=4$ then one of the components arising in $\overline{\overline{t h}}$ prime decomposition of $M$ must have virtually cyclic dimension $=4$. But for prime manifolds, we know that having virtually cyclic dimension $=4$ implies that the manifold is geometric (by Proposition 0.1), and looking at Table 2 we see the manifold must be crystallographic. This implies its fundamental group (itself a subgroup of $\Gamma$ ) contains a $\mathbb{Z}^{3}$ subgroup.

Statement (4). To complete the proof, let us now assume that $\Gamma$ is not virtually cyclic, nor a free product of virtually cyclic groups, nor has a $\mathbb{Z}^{3}$ subgroup. We will prove that $\operatorname{gd}(\Gamma)=3$. Let $M=P_{1} \# \cdots \# P_{k}$ with corresponding free splitting $\Gamma:=\Gamma_{1} * \cdots * \Gamma_{k}$. In view of Theorem 4.11, it suffices to show that all the prime manifolds $P_{i}$ in the decomposition satisfy $\underline{\underline{\operatorname{gd}}}\left(\Gamma_{i}\right) \leq 3$, and that at least one $P_{i}$ has $\underline{\underline{\operatorname{gd}}}\left(\Gamma_{i}\right)=3$.

First note that none of the $P_{i}$ can be crystallographic, since $\Gamma$ does not contain any $\mathbb{Z}^{3}$ subgroup. From Table 2, we see that if $P_{i}$ is prime, geometric, but not crystallographic, then $\operatorname{gd}\left(\Gamma_{i}\right) \leq 3$. On the other hand, if $P_{i}$ is not geometric, then from Proposition 9.1 it must have $\underline{\operatorname{gd}}\left(\Gamma_{i}\right)=3$. So we see that indeed all $\underline{\underline{\operatorname{gd}}}\left(\Gamma_{i}\right) \leq 3$.

Finally, if none of the $P_{i}$ have $\operatorname{gd}\left(\Gamma_{i}\right)=3$, then they must all satisfy $\operatorname{gd}\left(\Gamma_{i}\right) \leq 2$. Combining the results in Table 2 and Proposition $\left[.1\right.$ this can only happen if all the $\overline{\overline{P_{i}}}$ satisfy $\operatorname{gd}\left(\Gamma_{i}\right)=0$. From Table 2, this only occurs if all the $\Gamma_{i}$ are virtually cyclic, forcing $\Gamma$ to either be virtually cyclic (if there is only one prime factor) or to be a free product of virtually cyclic groups. But both of these statements are contradictions. We conclude that there must exist a $P_{i}$ with $\underline{\underline{\operatorname{gd}}}\left(\Gamma_{i}\right)=3$. Applying Theorem 4.11 gives us $\underline{\underline{\operatorname{gd}}}(\Gamma)=3$, and completes the proof of our main theorem. 
Remark 10.1. Looking through the proof of Theorem 1.1 above, we see that the exact same arguments also establish the geometric description given in Corollary 1.2 .

\section{REFERENCES}

[AFW15] Matthias Aschenbrenner, Stefan Friedl, and Henry Wilton. 3-manifold groups. EMS Series of Lectures in Mathematics. European Mathematical Society (EMS), Zürich, 2015.

[CFH06] Frank Conolly, Benjamin Fehrman, and Michael Hartglass. On the dimension of the virtually cyclic classifying space of a crystallographic group. arXiv:math/0610387, 2006.

[LM00] Wolfgang Lück and David Meintrup. On the universal space for group actions with compact isotropy. In Geometry and topology: Aarhus (1998), volume 258 of Contemp. Math., pages 293-305. Amer. Math. Soc., Providence, RI, 2000.

[LO07] Jean-François Lafont and Ivonne J. Ortiz. Relative hyperbolicity, classifying spaces, and lower algebraic K-theory. Topology, 46(6):527-553, 2007.

[LO09a] Jean-François Lafont and Ivonne J. Ortiz. Lower algebraic $K$-theory of hyperbolic 3 -simplex reflection groups. Comment. Math. Helv., 84(2):297-337, 2009.

[LO09b] Jean-François Lafont and Ivonne J. Ortiz. Splitting formulas for certain Waldhausen Nil-groups. J. Lond. Math. Soc. (2), 79(2):309-322, 2009.

[LW12] Wolfgang Lück and Michael Weiermann. On the classifying space of the family of virtually cyclic subgroups. Pure Appl. Math. Q., 8(2):497-555, 2012.

[Mor05] John W. Morgan. Recent progress on the Poincaré conjecture and the classification of 3-manifolds. Bull. Amer. Math. Soc. (N.S.), 42(1):57-78, 2005.

[MP02] Conchita Martínez-Pérez. A spectral sequence in Bredon (co)homology. J. Pure Appl. Algebra, 176(2-3):161173, 2002.

[Por08] Joan Porti. Geometrization of three manifolds and Perelman's proof. Rev. R. Acad. Cienc. Exactas Fís. Nat. Ser. A Mat. RACSAM, 102(1):101-125, 2008.

[Sco83] Peter Scott. The geometries of 3-manifolds. Bull. London Math. Soc., 15(5):401-487, 1983.

[Ser03] Jean-Pierre Serre. Trees. Springer Monographs in Mathematics. Springer-Verlag, Berlin, 2003. Translated from the French original by John Stillwell, Corrected 2nd printing of the 1980 English translation.

[Thu97] William P. Thurston. Three-dimensional geometry and topology. Vol. 1, volume 35 of Princeton Mathematical Series. Princeton University Press, Princeton, NJ, 1997. Edited by Silvio Levy.

Department of Mathematics, The Ohio State University, 100 Math Tower, 231 West 18th Avenue, Columbus, OH 43210-1174, USA

E-mail address: joecken@gmail.com

Department of Mathematics, The Ohio State University, 100 Math Tower, 231 West 18th Avenue, Columbus, OH 43210-1174, USA

E-mail address: jlafont@math.ohio-state.edu

Department of Mathematics, The Ohio State University, 100 Math Tower, 231 West 18th Avenue, Columbus, OH 43210-1174, USA

E-mail address: sanchezsaldana.1@osu.edu 